\title{
Distributing many points on spheres: minimal energy and designs
}

\author{
Johann S. Brauchart ${ }^{\mathrm{a}, \mathrm{b}, 1,2}$, Peter J. Grabner ${ }^{\mathrm{b}, 3}$ \\ ${ }^{a}$ School of Mathematics and Statistics, University of New South Wales, Sydney, NSW, 2052, Australia \\ ${ }^{b}$ Institut für Analysis und Computational Number Theory, Technische Universität Graz, Steyrergasse 30, \\ 8010 Graz, Austria
}

\begin{abstract}
This survey discusses recent developments in the context of spherical designs and minimal energy point configurations on spheres. The recent solution of the long standing problem of the existence of spherical $t$-designs on $\mathbb{S}^{d}$ with $\mathcal{O}\left(t^{d}\right)$ number of points by A. Bondarenko, D. Radchenko, and M. Viazovska attracted new interest to this subject. Secondly, D. P. Hardin and E. B. Saff proved that point sets minimising the discrete Riesz energy on $\mathbb{S}^{d}$ in the hypersingular case are asymptotically uniformly distributed. Both results are of great relevance to the problem of describing the quality of point distributions on $\mathbb{S}^{d}$, as well as finding point sets, which exhibit good distribution behaviour with respect to various quality measures.
\end{abstract}

\section{Dedicated to Edward B. Saff on the occasion of his $70^{\text {th }}$ birthday}

\section{Introduction}

The title of this survey is alluding to the fundamental paper by E. B. Saff and A. B. J. Kuijlaars [173], which discusses in large generality how to construct point sets on the sphere

$$
\mathbb{S}^{d}:=\left\{\mathbf{x} \in \mathbb{R}^{d+1} \mid\|\mathbf{x}\|=1\right\},
$$

which have "good distribution properties" with respect to various measures. That paper appeared in 1997 and motivated and initiated a fruitful direction of research. Evenly distributed point sets have many applications, the most prominent being numerical integration, approximation, interpolation, and sampling; each of these applications needs a different definition of what the word "evenly" should mean.

Email addresses: j.brauchart@tugraz.at (Johann S. Brauchart), peter.grabner@tugraz.at (Peter J. Grabner)

${ }^{1}$ This research was supported under Australian Research Council's Discovery Projects funding scheme (project number DP120101816).

${ }^{2}$ This author is supported by the Austrian Science Fund FWF projects F5510 (part of the Special Research Program (SFB) "Quasi-Monte Carlo Methods: Theory and Applications").

${ }^{3}$ This author is supported by the Austrian Science Fund FWF projects F5503 (part of the Special Research Program (SFB) "Quasi-Monte Carlo Methods: Theory and Applications") and W1230 (Doctoral Program "Discrete Mathematics"). 
The present survey on point distributions on the sphere is motivated by two rather recent important contributions, which both shed new light on the subject. The first was D. P. Hardin's and E. B. Saff's [116] proof that minimal energy point configurations on the sphere provide asymptotically uniformly distributed point sets for hypersingular Riesz potentials. This result has become popular under the name Poppy seed bagel theorem. This will be one subject of Section 3. The second breakthrough was A. V. Bondarenko's, D. Radchenko's, and M. S. Viazovska's [29] proof that spherical $t$-designs with $\mathcal{O}\left(t^{d}\right)$ number of points exist on $\mathbb{S}^{d}$. The definition of spherical designs, their properties, relevance, and occurrence in different contexts will be the subject of Section 2 .

Throughout the paper we use the notation $X_{N}=\left\{\mathbf{x}_{1}^{(N)}, \mathbf{x}_{2}^{(N)}, \ldots, \mathbf{x}_{N}^{(N)}\right\}$ for a set of $N$ points; $N$ will always denote the number of points, and the points $\mathbf{x}_{1}^{(N)}, \mathbf{x}_{2}^{(N)}, \ldots, \mathbf{x}_{N}^{(N)}$ usually depend on $N$. In order to keep the notation simple, we suppress this dependence.

Numerical computation of integrals over multidimensional domains is usually done by weighted sums of point evaluations of the function $f$ to be integrated. Since the sphere is a domain with very high symmetry, it is preferable to use equal weight integration methods,

$$
\int_{\mathbb{S} d} f(\mathbf{x}) \mathrm{d} \sigma_{d}(\mathbf{x}) \approx \frac{1}{N} \sum_{j=1}^{N} f\left(\mathbf{x}_{j}\right) .
$$

Here, and throughout the paper, we denote by $\sigma_{d}$ the normalised surface area measure on $\mathbb{S}^{d}$. For non-random collections of integration nodes, using the right-hand side of (1.1) as approximation for the integral is known as a Quasi-Monte Carlo (QMC) method. In order to obtain a good approximation of the integral by the sum, the point set $X_{N}$ should be well distributed over the whole sphere $\mathbb{S}^{d}$. The notion of good distribution of a point set is described in an intrinsic way in this context: the distribution is good, if the error for numerical integration is small for a certain set of functions. More precisely, sequence of node sets $\left(X_{N}\right)$ will be called asymptotically uniformly distributed, if the relation

$$
\lim _{N \rightarrow \infty} \frac{1}{N} \sum_{j=1}^{N} \mathbb{1}_{C}\left(\mathbf{x}_{j}\right)=\sigma_{d}(C)
$$

holds for all spherical caps $C$ (here and throughout the paper $\mathbb{1}_{C}$ denotes the indicator function of the set $C$ ). This is equivalent to

$$
\lim _{N \rightarrow \infty} \frac{1}{N} \sum_{j=1}^{N} f\left(\mathbf{x}_{j}\right)=\int_{\mathbb{S}^{d}} f(\mathbf{x}) \mathrm{d} \sigma_{d}(\mathbf{x})
$$

for all continuous functions $f: \mathbb{S}^{d} \rightarrow \mathbb{R}$ (see [137]).

Placing $N$ points on the sphere so that the least distance (or equivalently, the smallest angle) between two points,

$$
\delta\left(X_{N}\right):=\min _{1 \leq i<j \leq N}\left\|\mathbf{x}_{i}-\mathbf{x}_{j}\right\|
$$


is maximised is a further classical approach to obtain evenly distributed points, see [109]. This is the problem of best packing which has attracted attention for a long time in and outside of mathematics. For instance, the best packing problem on the sphere is attributed to P. M. L. Tammes [189], a botanist, who searched for an explanation of the surface structure of pollen grains.

A likewise geometric, but qualitatively different way of evenly distributing $N$ points is to cover the sphere with $N$ spherical caps of radius $r$, and to minimise $r$. The points are then chosen as the centres of these spherical caps. Equivalently, the size of the largest cap, which does not contain a point of $X_{N}$,

$$
\rho\left(X_{N}\right):=\max _{\mathbf{y} \in \mathbb{S}^{d}} \min _{1 \leq i \leq N}\left\|\mathbf{y}-\mathbf{x}_{i}\right\|
$$

should be as small as possible. This quantity is called the covering radius, and is also known as the mesh norm or the fill radius. This is the problem of best covering which originates from the realm of facility location problems where the farthest distance of a point on the sphere to the nearest point of $X_{N}$ (service distance) is minimised.

Sampling function values on the sphere (e.g., for approximation or interpolation by splines or radial basis functions) or exploring spatial directions in an efficient way requires again well distributed (but not necessary uniformly distributed) point sets on $\mathbb{S}^{d}$. In this context, the quality of a point set is measured differently: well distributed points are required to be dense in a quantifiable way. As the mesh norm arises in the error of approximation and good separation is generally associated with the "stability" of an approximation or interpolation method, one would prefer a quasi-uniform sequence of point sets $\left(X_{N}\right)$ with uniformly bounded mesh-separation ratio

$$
\gamma\left(X_{N}\right):=\frac{\rho\left(X_{N}\right)}{\delta\left(X_{N}\right)} .
$$

The mesh-separation ratio can be regarded as the "condition number" for the point set. Quasi-uniformity is a crucial property for a number of methods (see [96, 141, 177]).

\section{Spherical Designs}

Spherical designs were initially defined by P. Delsarte, J. M. Goethals, and J. J. Seidel [84] in the context of algebraic combinatorics on spheres. Since then, spherical designs have gained attraction in different areas of mathematics, ranging over number theory, geometry, algebraic and geometric combinatorics, and numerical analysis. We will give an account of these aspects in this section. For a survey on further developments in the context of spherical designs mainly from the point of view of algebraic combinatorics and number theory, we refer to the survey [15] by E. Bannai and E. Bannai.

\subsection{Definition}

A spherical $t$-design is a finite set of points $X_{N} \subset \mathbb{S}^{d}$, such that

$$
\frac{1}{N} \sum_{\mathbf{x} \in X_{N}} f(\mathbf{x})=\int_{\mathbb{S}^{d}} f(\mathbf{x}) \mathrm{d} \sigma_{d}(\mathbf{x})
$$


for all polynomials $f \in \mathbb{R}\left[x_{1}, \ldots, x_{d+1}\right]$ (restricted to the sphere $\mathbb{S}^{d}$ ) of total degree $\leq t$. This definition is equivalent to

$$
\sum_{(\mathbf{x}, \mathbf{y}) \in X_{N} \times X_{N}} P_{\ell}^{(d)}(\langle\mathbf{x}, \mathbf{y}\rangle)=0
$$

for $\ell=1, \ldots, t$; here $P_{\ell}^{(d)}$ denotes the Legendre polynomial of degree $\ell$ for the sphere $\mathbb{S}^{d}$ (see [156]). These polynomials are multiples of Gegenbauer polynomials $C_{\ell}^{\alpha}$ with $\alpha=\frac{d-1}{2}$ normalised so that $P_{\ell}^{(d)}(1)=1$. The equivalence of (2.2) and (2.1) is then an immediate consequence of the fact that the restrictions of polynomials to $\mathbb{S}^{d}$ are spanned by the harmonic polynomials (i.e., polynomials $p$ satisfying $\triangle_{d+1} p=0$ for the Laplace operator $\triangle_{d+1}$ in $\mathbb{R}^{d+1}$ ) and the addition theorem for spherical harmonics (see [156])

$$
\sum_{k=1}^{Z(d, \ell)} Y_{\ell, k}(\mathbf{x}) Y_{\ell, k}(\mathbf{y})=Z(d, \ell) P_{\ell}^{(d)}(\langle\mathbf{x}, \mathbf{y}\rangle) \text { with } Z(d, \ell):=\frac{2 \ell+d-1}{d-1}\left(\begin{array}{c}
\ell+d-2 \\
d-2
\end{array}\right)
$$

here and throughout this paper $Y_{\ell, k}(k=1, \ldots, Z(d, \ell))$ denotes a real orthonormal basis of the space of spherical harmonics of total degree $\ell$ with respect to the scalar product $\langle f, g\rangle:=\int_{\mathbb{S} d} f(\mathbf{x}) g(\mathbf{x}) \mathrm{d} \sigma_{d}(\mathbf{x})$. Condition (2.2) is then obtained by considering condition (2.1) for $f=Y_{\ell, k}$, squaring it, summing over $k$, and using (2.3).

There are two further characterisations of spherical $t$-designs, which show the connection of this concept to other areas of mathematics. It was observed in [148] that a spherical $2 t$-design $X_{N}$ with $N$ points gives an isometric embedding of $\ell_{2}^{d+1}$ into $\ell_{2 t}^{N}$, which comes from the identity

$$
\frac{1}{N} \sum_{\mathbf{x} \in X_{N}}\langle\mathbf{x}, \mathbf{a}\rangle^{2 t}=\frac{1 \cdot 3 \cdot 5 \cdots(2 t-1)}{(d+1)(d+2) \cdots(d+2 t-1)}\langle\mathbf{a}, \mathbf{a}\rangle^{t}
$$

which is valid for all $\mathbf{a} \in \mathbb{R}^{d+1}$. This identity is an immediate consequence of (2.2) and the expansion of $x^{2 t}$ as a sum of Legendre polynomials.

A spherical $t$-design $X_{N}=\left\{\mathbf{x}_{1}, \mathbf{x}_{2}, \ldots, \mathbf{x}_{N}\right\}$ is called rigid (see [14]), if there exists an $\varepsilon>0$, such that for all t-designs $X_{N}^{\prime}=\left\{\mathbf{x}_{1}^{\prime}, \mathbf{x}_{2}^{\prime}, \ldots, \mathbf{x}_{N}^{\prime}\right\}$ with $\left\|\mathbf{x}_{i}-\mathbf{x}_{i}^{\prime}\right\|<\varepsilon$ (for $i=1, \ldots, N)$ there exists a rotation $\eta \in \mathrm{SO}(d+1)$ such that $X_{N}^{\prime}=\eta X_{N}$.

It was observed in [104] in passing and later rediscovered in [185] that spherical $t$-designs can be characterised by a variational property. Let $p$ be a polynomial of degree $t$ given by

$$
p(z)=\sum_{\ell=1}^{t} a_{\ell} P_{\ell}^{(d)}(z) \quad \text { with } a_{\ell}>0 \text { for } \ell=1, \ldots, t .
$$

Then $X_{N}$ is a spherical $t$-design if and only if the energy functional $E_{p}\left(X_{N}\right)$ defined by

$$
E_{p}\left(X_{N}\right):=\sum_{(\mathbf{x}, \mathbf{y}) \in X_{N} \times X_{N}} p(\langle\mathbf{x}, \mathbf{y}\rangle)
$$


vanishes for $X_{N}$. Since the sum (2.5) is non-negative for all $X_{N}$, designs are minimisers of this sum. This was used in [185] to characterise designs as stationary points of $E_{p}\left(X_{N}\right)$.

In order to explain the connection to other questions of algebraic and geometric combinatorics on $\mathbb{S}^{d}$, we need the following notion. For a finite subset $X_{N}$ of $\mathbb{S}^{d}$, we define

$$
A\left(X_{N}\right):=\left\{\langle\mathbf{x}, \mathbf{y}\rangle \mid \mathbf{x}, \mathbf{y} \in X_{N}, \mathbf{x} \neq \mathbf{y}\right\}
$$

the set of mutual inner products of distinct points. For a given $A \subset[-1,1), X_{N}$ is called an $A$-code, if $A\left(X_{N}\right) \subset A$. Then, for instance, the problem of best packing of $N$ points on $\mathbb{S}^{d}$ can be formulated as finding the minimal $\beta$, such that there exists an $A$-code with $A=[-1, \beta]$. In particular, the determination of the kissing number, that is the maximum number of non-overlapping unit spheres that can touch another given unit sphere, is equivalent to finding the maximal cardinality of an $A$-code for $A=\left[-1, \frac{1}{2}\right]$ (see [157]).

\subsection{Relation to lattices}

Designs have a very interesting connection to the theory of lattices that we want to explain here. A lattice in $\mathbb{R}^{d}$ is a $\mathbb{Z}$-module

$$
\Lambda:=\mathbb{Z} \mathbf{v}_{1} \oplus \mathbb{Z} \mathbf{v}_{2} \oplus \cdots \oplus \mathbb{Z} \mathbf{v}_{d}
$$

where $\mathbf{v}_{1}, \ldots, \mathbf{v}_{d}$ is a basis of $\mathbb{R}^{d}$. For more detailed information on lattices, we refer to the book [78]. A lattice $\Lambda$ is called even, if all the squared norms $\|\mathbf{v}\|^{2}$ for $\mathbf{v} \in \Lambda$ are even. Even lattices can only exist if $d$ is divisible by 8 .

For any lattice $\Lambda$, the dual lattice is defined by

$$
\Lambda^{\#}:=\left\{\mathbf{x} \in \mathbb{R}^{d} \mid \forall \mathbf{v} \in \Lambda:\langle\mathbf{v}, \mathbf{x}\rangle \in \mathbb{Z}\right\}
$$

The set $\Lambda^{\#}$ is again a lattice. A lattice $\Lambda$ is called unimodular, if $\Lambda^{\#}=\Lambda$. For an even unimodular lattice $\Lambda$, the $\vartheta$-series

$$
\vartheta_{\Lambda}(\tau)=\sum_{\mathbf{v} \in \Lambda} e^{\pi i\|\mathbf{v}\|^{2} \tau}, \quad \tau \in \mathbb{C}, \quad \operatorname{Im}(\tau)>0
$$

is a modular form of weight $d / 2$; i.e., the transformation formula

$$
\vartheta_{\Lambda}(-1 / \tau)=\tau^{d / 2} \vartheta_{\Lambda}(\tau)
$$

holds for all $\tau$ with $\operatorname{Im}(\tau)>0$. This follows from an application of Poisson's summation formula.

The theory of modular forms (see [8]) yields that for a form $f$ of weight $d / 2$, given by

$$
f(\tau)=\sum_{n=0}^{\infty} a_{n} e^{2 \pi i n \tau}
$$


at least one of the coefficients $a_{n}$ for $n=1, \ldots, 2+2\left\lfloor\frac{d}{24}\right\rfloor$ has to be non-zero. This implies that every even unimodular lattice $\Lambda$ contains a non-zero vector $\mathbf{v}$ with $\|\mathbf{v}\| \leq 2+2\left\lfloor\frac{d}{24}\right\rfloor$. If the shortest non-zero vector $\mathbf{v}$ in $\Lambda$ satisfies $\|\mathbf{v}\|^{2}=2+2\left\lfloor\frac{d}{24}\right\rfloor$, then the lattice is called extremal.

For a homogeneous polynomial $p$ of degree $j \geq 1$, which is also harmonic $\left(\triangle_{d} p=0\right)$, and an even unimodular lattice $\Lambda$, the series

$$
\vartheta_{\Lambda, p}(\tau)=\sum_{\mathbf{v} \in \Lambda} p(\mathbf{v}) e^{\pi i\|\mathbf{v}\|^{2} \tau}, \quad \operatorname{Im}(\tau)>0,
$$

is a modular form of weight $d / 2+j$. It is immediate from the symmetry of $\Lambda$ and the homogeneity of $p$ that $\vartheta_{\Lambda, p}=0$, if $j$ is odd. If $j$ is even, the series $\vartheta_{\Lambda, p}$ is a cusp form; i.e.,

$$
\lim _{\operatorname{Im}(\tau) \rightarrow \infty} \vartheta_{\Lambda, p}(\tau)=0
$$

since $p(\mathbf{0})=0$.

From the theory of modular forms (see [8]) it is known that there is a unique cusp form $\Delta(\tau)$ (this standard notation "Delta" is not to be confused with the Laplace operator $\triangle$ "triangle") of weight 12 having an expansion of the form

$$
\Delta(\tau)=e^{2 \pi i \tau}+\sum_{n=2}^{\infty} a_{n} e^{2 \pi i n \tau} .
$$

It follows from degree considerations that for an even, unimodular, extremal lattice $\Lambda$ and a homogeneous, harmonic polynomial $p$ of degree $j \geq 1$,

$$
\vartheta_{\Lambda, p}(\tau)=\Delta(\tau)^{1+\left\lfloor\frac{d}{24}\right\rfloor} f(\tau),
$$

where $f(\tau)$ is a modular form of weight

$$
\frac{d}{2}-12\left\lfloor\frac{d}{24}\right\rfloor+j-12 .
$$

If this weight is negative, then $f$ has to vanish identically, since there are no modular forms of negative weight. Consider $d=24 m+k$ with $k \in\{0,8,16\}$ and insert this into the above equation. This yields $k / 2+j-12$ for the weight of $f$. For $k=0,8$, and 16, this yields negative weights for $j \leq 11,7$, and 3 , respectively, which shows that for all shells of the corresponding lattices the sum

$$
\sum_{\|\mathbf{v}\|^{2}=n} p(\mathbf{v})=n^{j / 2} \sum_{\|\mathbf{v}\|^{2}=n} p(\mathbf{v} / \sqrt{n})=0
$$

for all homogeneous harmonic polynomials of degree $1 \leq j \leq 11,7,3$, respectively. Summing up, we have proved the following theorem.

Theorem 2.1 ([195]). Let $\Lambda$ be an extremal even unimodular lattice in $\mathbb{R}^{d}$ with $d=24 m+$ $k$ for $k=0,8,16$. Then any non-empty shell of $\Lambda$ defines a $j$-design $n^{-\frac{1}{2}}\left\{\mathbf{v} \in \Lambda \mid\|\mathbf{v}\|^{2}=n\right\}$ for $j=11-k / 2$.

For further developments in the context of designs, lattices, modular forms, and algebraic codes, we refer to [10, 12, 15, 80, 158, 159]. 


\subsection{Lower bounds}

In 84] a linear programming method has been developed, which allows to produce lower bounds for the number of points $N$ of a spherical $t$-design. This method has been applied successfully to related questions, such as find the minimal cardinality of $A\left(X_{N}\right)$ for sets $X_{N}$ with $N$ points.

We give a short explanation of this powerful method, that has been applied with great success to several problems of discrete geometry, the most prominent being the solution of Kepler's conjecture by T. Hales [110]. Since we use the method only on the sphere, we restrict the description to this case here. Let $f:[-1,1] \rightarrow \mathbb{R}^{+}$be a continuous positive function given by its expansion in terms of Legendre polynomials; i.e.,

$$
f(x)=\sum_{n=0}^{\infty} \widehat{f}(n) Z(d, n) P_{n}^{(d)}(x) .
$$

If the coefficients $\widehat{f}(n)$ are non-positive for $n>t$, then any $t$-design $X_{N}$ has to have cardinality $N \geq f(1) / \widehat{f}(0)$. The proof of this fact is given by the relations

$$
\begin{aligned}
f(1) N & \leq \sum_{(\mathbf{x}, \mathbf{y}) \in X_{N} \times X_{N}} f(\langle\mathbf{x}, \mathbf{y}\rangle) \\
& =\widehat{f}(0)(N)^{2}+\sum_{n>t} \widehat{f}(n) Z(d, n) \sum_{(\mathbf{x}, \mathbf{y}) \in X_{N} \times X_{N}} P_{n}^{(d)}(\langle\mathbf{x}, \mathbf{y}\rangle) \\
& \leq \widehat{f}(0) N^{2} .
\end{aligned}
$$

The first inequality is a consequence of the positivity of $f$, the equality uses the characterisation of $t$-designs by (2.2), and the second inequality uses that $\widehat{f}(n) \leq 0$ for $n>t$ and the non-negativity of the double sums $\sum_{(\mathbf{x}, \mathbf{y}) \in X_{N} \times X_{N}} P_{n}^{(d)}(\langle\mathbf{x}, \mathbf{y}\rangle)$. Equality can only occur, if $f(x)=0$ for all $x \in A\left(X_{N}\right)$ and $\widehat{f}(n)=0$ for $n>t$; i.e., $f$ is a polynomial of degree $\leq t$.

In [84] a polynomial $p_{t}$ of degree $t$ was constructed, which gives the lower bound

$$
N \geq \begin{cases}\left(\begin{array}{c}
d+t / 2 \\
d
\end{array}\right)+\left(\begin{array}{c}
d+t / 2-1 \\
d
\end{array}\right) & \text { for } t \text { even } \\
2\left(\begin{array}{c}
d+(t-1) / 2 \\
d
\end{array}\right) & \text { for } t \text { odd }\end{cases}
$$

This polynomial actually provides the best possible lower bound that can be obtained by polynomial functions $f$. Designs attaining this lower bound are called tight. It was shown in [16, 17] that tight designs only exist for $d=1$ and all $t$, or finitely many values of $t$ if $d \geq 2$. The proof uses the fact that for a tight spherical $t$-design $X_{N}$, the corresponding set $A\left(X_{N}\right)$ has to consist exactly of the zeros of the polynomial $p_{t}$ constructed in [84]. Galois theory is then used to derive a contradiction, if $t$ is large enough. The same fact about $A\left(X_{N}\right)$ shows that tight designs are rigid (see [14]). Notice that the bound is of order $t^{d}$. Later, V. A. Yudin [199] considered a wider class of functions than polynomials. He constructed a function $f$, which allowed for a considerable improvement of the lower bound. The bounds for spherical designs he gives are again of order $t^{d}$, but they are 
larger by a factor depending on $d$. This factor is exponential in $d$. For $d=2$, the gain is asymptotically about $7 \%$.

We give a short description of Yudin's construction. The function $f$ is obtained as the spherical convolution of two positive functions $F$ and $G$, which ensures the positivity of $f$. The spherical convolution of two functions $F$ and $G$ is given by

$$
F \star G(\langle\mathbf{x}, \mathbf{y}\rangle):=\int_{\mathbb{S}^{d}} F(\langle\mathbf{x}, \mathbf{z}\rangle) G(\langle\mathbf{z}, \mathbf{y}\rangle) \mathrm{d} \sigma_{d}(\mathbf{z}) .
$$

In order to obtain the required sign change of the Laplace-Fourier coefficients of $f$, the function $G$ is chosen as

$$
G(x)=\left(1-x^{2}\right)^{1-\frac{d}{2}}\left(\left(1-x^{2}\right)^{\frac{d}{2}} F^{\prime}(x)\right)^{\prime}+(t+1)(t+d) F(x),
$$

which is motivated by the fact that the Legendre polynomials are eigenfunctions of the differential operator $L$ defined by

$$
L F(x):=\left(1-x^{2}\right)^{1-\frac{d}{2}}\left(\left(1-x^{2}\right)^{\frac{d}{2}} F^{\prime}(x)\right)^{\prime} .
$$

If $F$ is expressed in terms of its Laplace-Fourier expansion

$$
F(x)=\sum_{n=0}^{\infty} \widehat{F}(n) Z(d, n) P_{n}^{(d)}(x)
$$

then the function $G$ is given by

$$
G(x)=\sum_{n=0}^{\infty}(t+1-n)(n+t+d) \widehat{F}(n) Z(d, n) P_{n}^{(d)}(x) .
$$

Hence the spherical convolution is given as

$$
f(x)=F \star G(x)=\sum_{n=0}^{\infty}(t+1-n)(n+t+d) \widehat{F}(n)^{2} Z(d, n) P_{n}^{(d)}(x),
$$

which has the required sign-change in its Laplace-Fourier coefficients. The problem of maximising the quotient $f(1) / \widehat{f}(0)=f(1) / \widehat{F}(0)^{2}$ turns out to be a variational problem for $F$ :

$$
\begin{aligned}
f(1) & =\frac{\omega_{d-1}}{\omega_{d}} \int_{-1}^{1} F(x) G(x)\left(1-x^{2}\right)^{d / 2-1} \mathrm{~d} x \\
& =\frac{\omega_{d-1}}{\omega_{d}} \int_{-1}^{1}\left(-\left(1-x^{2}\right) F^{\prime}(x)^{2}+(t+1)(t+d) F(x)^{2}\right)\left(1-x^{2}\right)^{d / 2-1} \mathrm{~d} x
\end{aligned}
$$

must be maximised subject to the condition

$$
\widehat{F}(0)=\frac{\omega_{d-1}}{\omega_{d}} \int_{-1}^{1} F(x)\left(1-x^{2}\right)^{d / 2-1} \mathrm{~d} x=1 .
$$


The symbol $\omega_{d}$ denotes the surface area of $\mathbb{S}^{d}$ and it satisfies

$$
\int_{-1}^{1}\left(1-x^{2}\right)^{d / 2-1} \mathrm{~d} x=\frac{\omega_{d}}{\omega_{d-1}}=\frac{\sqrt{\pi} \Gamma(d / 2)}{\Gamma((d+1) / 2)} .
$$

Furthermore, $f$ has to be non-negative on $[-1,1]$. This is achieved by assuming that $F$ and $G$ are both non-negative. The solution of this variational problem with the additional condition on the sign of $F$ is then given by

$$
F(x)= \begin{cases}P_{t+1}^{(d)}(x)-P_{t+1}^{(d)}\left(\alpha_{t}\right) & \text { for } \alpha_{t} \leq x \leq 1, \\ 0 & \text { for }-1 \leq x \leq \alpha_{t},\end{cases}
$$

where $\alpha_{t}$ is the largest zero of $\frac{d}{d x} P_{t+1}^{(d)}(x)$. In this case $G$ is a piecewise constant function. Putting everything together yields the lower bound

$$
N \geq \frac{\int_{-1}^{1}\left(1-x^{2}\right)^{d / 2-1} \mathrm{~d} x}{\int_{\alpha_{t}}^{1}\left(1-x^{2}\right)^{d / 2-1} \mathrm{~d} x}=\frac{1}{\sigma_{d}\left(\left\{\mathbf{y} \in \mathbb{S}^{d} \mid\langle\mathbf{y}, \mathbf{x}\rangle \geq \alpha_{t}\right\}\right)} \gg_{d} t^{d}
$$

for a $t$-design $X_{N}$. For a more detailed exposition, we refer to [199]. Even if the obtained function $f$ gives a better value for the lower bound than the polynomial given in [84], the technical requirement of non-negativity of $F$ seems to leave room for further improvement.

A similar construction for a function $f$ is used in [73] to obtain linear programming bounds for the packing density of spheres in $\mathbb{R}^{d}$. The function obtained there has similar features; in particular, it is supported on a short interval. In [73, Section 5] it is mentioned that the function constructed by this convolution method does not produce optimal bounds (see [72]). The reason seems to be exactly the non-negativity requirement on the corresponding function $F$. Thus there is reason to believe that Yudin's lower bound for the cardinality of $t$-designs can still be improved.

\subsection{Existence}

On the other hand, the question of the existence of spherical $t$-designs has been answered affirmatively. First, a rather general result obtained in [181] shows that, for given $t$ and large enough $N$, there exists a $t$-design with $N$ points. Actually, the result given in [181] is more general: given a path connected topological space $\Omega$ and a finite measure $\mu$ that charges every non-empty open set, then for any finite set of continuous real valued functions $f_{1}, \ldots, f_{t}$ there exists an $N_{0}$, such that for every $N>N_{0}$ there exists a set $X_{N} \subset \Omega$, such that

$$
\frac{1}{N} \sum_{x \in X_{N}} f_{j}(x)=\frac{1}{\mu(\Omega)} \int_{\Omega} f_{j}(x) \mathrm{d} \mu(x)
$$

for $j=1, \ldots, t$. The result gives a bound for the number of points needed by geometric quantities defined in terms of $\Omega$ and the functions $f_{1}, \ldots, f_{t}$. These quantities are very difficult to compute even in the special case of the sphere. 
In order to discuss the question of existence of spherical designs further, we introduce two quantities:

$$
\begin{aligned}
N(t, d) & :=\min \left\{N \mid \exists X_{N} \subset \mathbb{S}^{d}: X_{N} \text { is a } t \text {-design }\right\}, \\
N^{*}(t, d) & :=\min \left\{K \mid \forall N \geq K: \exists X_{N} \subset \mathbb{S}^{d}: X_{N} \text { is a } t \text {-design }\right\} .
\end{aligned}
$$

By definition it is clear that $N^{*}(t, d) \geq N(t, d)$.

Only very recently, the long standing problem of the existence of spherical $t$-designs with $\mathcal{O}\left(t^{d}\right)$ points was answered affirmatively by A. V. Bondarenko, D. Radchenko, and M. S. Viazovska [29]. They proved that $N^{*}(t, d)=\mathcal{O}\left(t^{d}\right)$ with an explicit, but large implied constant. The proof puts the existence of spherical $t$-designs with $N=\mathcal{O}\left(t^{d}\right)$ in the context of Brouwer's degree theorem: Let $\mathcal{P}_{t}$ denote the Hilbert space of polynomials $P$ of total degree $\leq t$ with

$$
\int_{\mathbb{S}^{d}} P(\mathbf{x}) \mathrm{d} \sigma_{d}(\mathbf{x})=0
$$

equipped with the scalar product

$$
\langle P, Q\rangle=\int_{\mathbb{S}^{d}} P(\mathbf{x}) Q(\mathbf{x}) \mathrm{d} \sigma_{d}(\mathbf{x}) .
$$

Then for every $\mathbf{x} \in \mathbb{S}^{d}$ there exists a polynomial $G_{\mathbf{x}}$ such that

$$
\left\langle G_{\mathbf{x}}, Q\right\rangle=Q(\mathbf{x})
$$

for all $Q \in \mathcal{P}_{t}$. A set of points $X_{N}$ then is a spherical $t$-design, if

$$
G_{\mathbf{x}_{1}}+\cdots+G_{\mathbf{x}_{N}} \equiv 0 .
$$

Then continuous maps $\mathbf{x}_{i}: \mathcal{P}_{t} \rightarrow \mathbb{S}^{d}$ are constructed, which in turn define the map $f$ : $\mathcal{P}_{t} \rightarrow \mathcal{P}_{t}$ by

$$
f(P):=G_{\mathbf{x}_{1}(P)}+\cdots+G_{\mathbf{x}_{N}(P)} .
$$

Then for any $P \in \mathcal{P}_{t}$ the identity

$$
\langle P, f(P)\rangle=\sum_{i=1}^{N} P\left(\mathbf{x}_{i}(P)\right)
$$

holds. A polynomial $\tilde{P}$ with $f(\tilde{P})=0$ then gives a spherical $t$-design $\left\{\mathbf{x}_{1}(\tilde{P}), \ldots, \mathbf{x}_{N}(\tilde{P})\right\}$. The construction of the maps $\mathbf{x}_{i}$ is the crucial part of the proof. Starting with points $\left\{\mathbf{x}_{1}(0), \ldots, \mathbf{x}_{N}(0)\right\}$ in the parts of an equal area partition of the sphere, the maps $\mathbf{x}_{i}$ are defined by an intricate geometric procedure. The proof finishes by considering the set

$$
\Omega=\left\{P \in \mathcal{P}_{t} \mid \int_{\mathbb{S}^{d}}\|\nabla P(\mathbf{x})\| \mathrm{d} \sigma_{d}(\mathbf{x})<1\right\}
$$


and observing that the construction of $\mathbf{x}_{i}$ yields

$$
\langle P, f(P)\rangle>0
$$

for $P \in \partial \Omega$. This last inequality is verified by an application of a spherical version of the Marcinkiewicz-Zygmund inequality. In this step the precise choice of the number $N$ of points in relation to $t$ is significant. The Brouwer degree theorem gives the existence of a point $\tilde{P}$ with $f(\tilde{P})=0$, which the yields the desired spherical $t$-design $\left\{\mathbf{x}_{1}(\tilde{P}), \ldots, \mathbf{x}_{N}(\tilde{P})\right\}$.

In a recent paper [30], the same authors showed that there exist well separated $t$-designs with optimal order of the number of points:

Theorem 2.2. Let $d \geq 2$. Then there exist positive constants $C_{d}$ and $\beta_{d}$ such that for every $N \geq C_{d} t^{d}$ there exists a $t$-design $X_{N}$ and

$$
\|\mathbf{x}-\mathbf{y}\| \geq \beta_{d} N^{-1 / d} \quad \text { for all } \mathbf{x}, \mathbf{y} \in X_{N} \text { with } \mathbf{x} \neq \mathbf{y} .
$$

The proof is a refinement of their original proof by keeping control on the distance of distinct points.

\subsection{Numerical results}

Besides the theoretical investigation of the existence of spherical designs, several attempts were made to compute lists of $t$-designs for moderately large values of $t$ and $N$. Since most of these computations have been done for $d=2$, we will restrict to that case in this section.

A first list of $t$-designs for $t \leq 21$ was provided by R. H. Hardin and N. J. A. Sloane (see [120, 121, 122]). Their list is still available on the web [123]. These numerical computations, as well as those performed in [185], seem to suggest that $N(t, 2)$ is close to $\frac{1}{2}(t+1)^{2}$. Furthermore, X. Chen and R. S. Womersley found spherical $t$-designs by numerical computations for $t \leq 100$ (see [68]). Their computations seem to indicate that there exist $t$-designs with less than $(t+1)^{2}$ points. Later X. Chen, A. Frommer, and B. Lang [67] used interval arithmetic to prove that there exist spherical $t$-designs with $(t+1)^{2}$ points for $1 \leq t \leq 100$. Recently, M. Gräf and D. Potts [105, 106] derived a new method based on fast Fourier transform, which allows to find $t$-designs numerically with high precision for values of $t$ up to 1000. They also provide their results on the web [107]. Again these numerical results show that $N(t, 2)$ is close to $\frac{1}{2}(t+1)^{2}$ for small values of $t$.

\subsection{Designs and uniform distribution}

It was observed independently in [104] and in [133] that spherical designs provide welldistributed point sets on the sphere.

The spherical cap discrepancy of a point set $X_{N}$ of $N$ points is given by

$$
D\left(X_{N}\right):=\sup _{\substack{\mathbf{y} \in \mathbb{S}^{d} \\ \varphi \in[0, \pi]}}\left|\frac{1}{N} \sum_{j=1}^{N} \mathbb{1}_{C(\mathbf{y}, \varphi)}\left(\mathbf{x}_{j}\right)-\sigma_{d}(C(\mathbf{y}, \varphi))\right| ;
$$


Table 1: The lower bounds for the number of points of a $t$-design derived in [84] (DGS $(t))$ and in 199] $(\mathrm{Y}(t))$ compared to the number of points of $t$-designs $N(t, 2)$ obtained in [106] (as provided on the website [107]).

\begin{tabular}{|l|c|c|c|c|c|c|c|c|c|c|c|c|c|c|c|}
\hline$t$ & 5 & 7 & 9 & 10 & 20 & 30 & 40 & 50 & 60 & 70 & 80 & 90 & 100 & 114 & 124 \\
\hline \hline DGS $(t)$ & 12 & 20 & 30 & 36 & 121 & 256 & 441 & 676 & 961 & 1296 & 1681 & 2116 & 2601 & 3364 & 3969 \\
\hline $\mathrm{Y}(t)$ & 12 & 20 & 31 & 37 & 127 & 271 & 470 & 723 & 1031 & 1394 & 1810 & 2282 & 2808 & 3635 & 4292 \\
\hline$N(t, 2)$ & 12 & 24 & 48 & 60 & 216 & 480 & 840 & 1296 & 1860 & 2520 & 3276 & 4140 & 5100 & 6612 & 7812 \\
\hline$\left\lfloor\frac{(t+1)^{2}}{2}\right\rfloor$ & 18 & 32 & 50 & 60 & 220 & 480 & 840 & 1300 & 1860 & 2520 & 3280 & 4140 & 5100 & 6612 & 7812 \\
\hline
\end{tabular}

the supremum is extended over all spherical caps

$$
C(\mathbf{y}, \varphi):=\left\{\mathbf{x} \in \mathbb{S}^{d} \mid\langle\mathbf{x}, \mathbf{y}\rangle>\cos (\varphi)\right\}
$$

and measures the maximum deviation between the empirical distribution of the point set $X_{N}$ from uniform distribution. In [102] the estimate

$$
D\left(X_{N}\right) \leq \frac{C_{1}(d)}{M}+\sum_{\ell=1}^{M} \frac{C_{2}(d)}{\ell} \sum_{k=1}^{Z(d, \ell)}\left|\frac{1}{N} \sum_{j=1}^{N} Y_{\ell, k}\left(\mathbf{x}_{j}\right)\right|
$$

was proved; here $M$ is an arbitrary positive integer, and $C_{1}(d)$ and $C_{2}(d)$ are explicit constants depending only on the dimension $d$. A similar inequality was later given in [144]. The inequality (2.17) resembles the classical Erdős-Turán-Koksma inequality estimating the Euclidean discrepancy of a point set in $[0,1]^{d}$ in terms of trigonometric sums (see [137]). In [135], N. M. Korobov introduced good lattice points $\left(g_{1}, \ldots, g_{d}\right) \in \mathbb{Z}^{d}$ by the requirement that the point set $\left\{\left(\left\{\frac{j g_{1}}{N}\right\}, \ldots,\left\{\frac{j g_{d}}{N}\right\}\right) \mid j=0, \ldots, N-1\right\}$ has small discrepancy.

In [104] spherical $t$-designs were regarded as spherical analogues of good lattice points, in the sense that the estimate (2.17) becomes particularly simple when applied to a $t$-design and choosing the parameter $M$ to be $t$ : the estimate then reduces to

$$
D\left(X_{N}\right) \leq \frac{C_{1}(d)}{t}
$$

Similarly, for a continuous function $f: \mathbb{S}^{d} \rightarrow \mathbb{R}$ satisfying the Lipschitz-condition $\mid f(\mathbf{x})$ $f(\mathbf{y}) \mid \leq C_{f} \arccos (\langle\mathbf{x}, \mathbf{y}\rangle)$, the estimate

$$
\left|\frac{1}{N} \sum_{\mathbf{x} \in X_{N}} f(\mathbf{x})-\int_{\mathbb{S}^{d}} f(\mathbf{x}) \mathrm{d} \sigma_{d}(\mathbf{x})\right| \leq C_{f}\left(6 \frac{d}{M}+\pi \sum_{\ell=1}^{2 M} \sum_{k=1}^{Z(d, \ell)}\left|\frac{1}{N} \sum_{\mathbf{x} \in X_{N}} Y_{\ell, k}(\mathbf{x})\right|\right)
$$

was shown in the same paper. Again, taking $X_{N}$ to be a $2 t$-design and $M=t$ gives an estimate $6 d C_{f} / t$ for the integration error.

J. Korevaar and J. L. H. Meyers [133] take a potential theoretic point of view. In their papers [133, 134] they conjecture that $N(t, d)=\mathcal{O}\left(t^{d}\right)$, which was finally proved in [29]. 
Let $\mu_{N}:=\frac{1}{N} \sum_{\mathbf{x} \in X_{N}} \delta_{\mathbf{x}}$ be the discrete equal weight distribution supported on $X_{N} \subset \mathbb{S}^{d}$. Then the deviation of $\mu_{N}$ and the equilibrium measure $\sigma_{d}$ is measured by the deviation of the potential

$$
U_{d-1}^{\mu_{N}}(\mathbf{x})=\frac{1}{N} \sum_{\mathbf{y} \in X_{N}} \frac{1}{\|\mathbf{x}-\mathbf{y}\|^{d-1}}=\int_{\mathbb{S}^{d}} \frac{1}{\|\mathbf{x}-\mathbf{y}\|^{d-1}} \mathrm{~d} \mu_{N}(\mathbf{y})
$$

from the equilibrium potential

$$
U_{d-1}^{\sigma_{d}}(\mathbf{x})=\int_{\mathbb{S}^{d}} \frac{1}{\|\mathbf{x}-\mathbf{y}\|^{d-1}} \mathrm{~d} \sigma_{d}(\mathbf{y})=1
$$

for $\|\mathbf{x}\| \leq r<1$.

Taking a spherical $t$-design $X_{N}$ and using the identity (see [149])

$$
\frac{1}{\|r \mathbf{x}-\mathbf{y}\|^{d-1}}=\sum_{n=0}^{\infty}\left(\begin{array}{c}
n+d-2 \\
d-2
\end{array}\right) P_{n}^{(d)}(\langle\mathbf{x}, \mathbf{y}\rangle) r^{n}
$$

we obtain

$$
U_{d-1}^{\mu_{N}}(r \mathbf{x})-1=\sum_{n=t+1}^{\infty}\left(\begin{array}{c}
n+d-2 \\
d-2
\end{array}\right) r^{n} \frac{1}{N} \sum_{\mathbf{y} \in X_{N}} P_{n}^{(d)}(\langle\mathbf{x}, \mathbf{y}\rangle) .
$$

Estimating the right hand side by $\left|P_{n}^{(d)}(\cdot)\right| \leq 1$, we obtain

$$
\left|U_{d-1}^{\mu_{N}}(r \mathbf{x})-1\right| \leq \sum_{n=t+1}^{\infty}\left(\begin{array}{c}
n+d-2 \\
d-2
\end{array}\right) r^{n}
$$

The sum on the right hand side can be expressed in closed form.

$$
\begin{aligned}
& \sum_{n=t+1}^{\infty}\left(\begin{array}{c}
n+d-2 \\
d-2
\end{array}\right) r^{n} \\
& \quad=(d-1)\left(\begin{array}{c}
t+d-1 \\
d-1
\end{array}\right) \frac{1}{(1-r)^{d-1}} \int_{0}^{r}(1-\rho)^{d-2} \rho^{t} \mathrm{~d} \rho \\
& \quad=\frac{r^{t+1}}{(1-r)^{d-1}}(d-1)\left(\begin{array}{c}
t+d-1 \\
d-1
\end{array}\right) \sum_{\ell=0}^{d-2}\left(\begin{array}{c}
d-2 \\
\ell
\end{array}\right)(-1)^{\ell} \frac{r^{\ell}}{t+\ell+1}
\end{aligned}
$$

which can be proved by multiplying with $(1-r)^{d-1}$ and differentiating. Estimating the integral in the second line by $r^{t+1} /(t+1)$ we get the estimate

$$
\left|U_{d-1}^{\mu_{N}}(r \mathbf{x})-1\right| \leq\left(\begin{array}{c}
t+d-1 \\
d-2
\end{array}\right) \frac{r^{t+1}}{(1-r)^{d-1}}
$$

valid for any $t$-design $X_{N}, \mathbf{x} \in \mathbb{S}^{d}$, and $0 \leq r<1$. This, together with the fact that $N(t, d)=\mathcal{O}\left(t^{d}\right)$, generalises [133, Theorem 2.2] to arbitrary dimension. 


\subsection{Applications to numerical integration on $\mathbb{S}^{d}$}

Equal weight quadrature formulas like spherical design QMC methods are especially useful for integrating functions taken from suitably defined Sobolev spaces on $\mathbb{S}^{d}$. These spaces are reproducing kernel Hilbert spaces, which makes the study of the worst case error in integration particularly simple and transparent (see [162]).

In order to describe the results on numerical integration in more detail, we give a precise definition of the function spaces. The negative Laplace-Beltrami operator $-\triangle_{d}^{*}$ on $\mathbb{S}^{d}$ has the eigenvalues $\lambda_{\ell}:=\ell(\ell+d-1), \ell \in \mathbb{N}_{0}$. The space of eigenfunctions for the eigenvalue $\lambda_{\ell}$ is spanned by the spherical harmonics $Y_{\ell, k}$ for $k=1, \ldots, Z(d, \ell)$. Since the eigenfunctions of $-\triangle_{d}^{*}$ form a complete function system, every function $f \in L^{2}\left(\mathbb{S}^{d}\right)$ can be represented by its Laplace-Fourier series expansion

$$
f(\mathbf{x})=\sum_{\ell=0}^{\infty} \sum_{k=1}^{Z(d, \ell)} \widehat{f}_{\ell, k} Y_{\ell, k}(\mathbf{x})
$$

where the Laplace-Fourier coefficients are given by

$$
\widehat{f}_{\ell, k}=\int_{\mathbb{S}^{d}} f(\mathbf{x}) Y_{\ell, k}(\mathbf{x}) \mathrm{d} \sigma_{d}(\mathbf{x})
$$

The series (2.23) has to be interpreted in the $L^{2}$-sense. Furthermore, Parseval's identity

$$
\sum_{\ell=0}^{\infty} \sum_{k=1}^{Z(d, \ell)}\left|\widehat{f}_{\ell, k}\right|^{2}=\int_{\mathbb{S}^{d}}|f(\mathbf{x})|^{2} \mathrm{~d} \sigma_{d}(\mathbf{x})=\|f\|_{2}^{2}
$$

and the Funk-Hecke formula

$$
\sum_{k=1}^{Z(d, \ell)} \widehat{f}_{\ell, k} Y_{\ell, k}(\mathbf{x})=Z(d, \ell) \int_{\mathbb{S}^{d}} f(\mathbf{y}) P_{\ell}^{(d)}(\langle\mathbf{x}, \mathbf{y}\rangle) \mathrm{d} \sigma_{d}(\mathbf{y})
$$

hold. For more details on the harmonic analysis on $\mathbb{S}^{d}$, we refer to [156].

In the following we adopt the notation of [61]. For $s \geq 0$, we define the space

$$
H^{s}\left(\mathbb{S}^{d}\right):=\left\{\left.f \in L^{2}\left(\mathbb{S}^{d}\right)\left|\sum_{\ell=0}^{\infty}(1+\ell)^{2 s} \sum_{k=1}^{Z(d, \ell)}\right| \widehat{f}_{\ell, k}\right|^{2}<\infty\right\} .
$$

From this definition, it is clear that the "sequence of weights" $\left((1+\ell)^{2 s}\right)$ can be replaced by any other comparable sequence $\left(w_{\ell}\right)$, in the sense of

$$
\exists C_{1}, C_{2}>0: \forall \ell \in \mathbb{N}_{0}: C_{1}(1+\ell)^{2 s} \leq w_{\ell} \leq C_{2}(1+\ell)^{2 s} .
$$

We define a norm on $H^{s}\left(\mathbb{S}^{d}\right)$ by

$$
\|f\|_{H^{s}}:=\left(\sum_{\ell=0}^{\infty}(1+\ell)^{2 s} \sum_{k=1}^{Z(d, \ell)}\left|\widehat{f}_{\ell, k}\right|^{2}\right)^{1 / 2} .
$$


From the definition it is clear that the spaces $H^{s}\left(\mathbb{S}^{d}\right)$ are getting smaller as the index of smoothness $s$ increases. Furthermore, the Sobolev embedding theorem ensures that $H^{s}\left(\mathbb{S}^{d}\right)$ embeds continuously into $C^{k}\left(\mathbb{S}^{d}\right)$ when $s>k+d / 2$; in particular, $H^{s}\left(\mathbb{S}^{d}\right)$ embeds continuously into $C\left(\mathbb{S}^{d}\right)$ when $s>d / 2$.

As a consequence of the embedding of $H^{s}\left(\mathbb{S}^{d}\right)$ into $C\left(\mathbb{S}^{d}\right)$ for $s>d / 2$, point evaluation of a function is a continuous functional, which can be represented as a scalar product by the Riesz representation theorem. This ensures the existence of a reproducing kernel given by

$$
\mathrm{K}^{(s)}(\langle\mathbf{x}, \mathbf{y}\rangle):=\sum_{\ell=0}^{\infty}(1+\ell)^{-2 s} Z(d, \ell) P_{\ell}^{(d)}(\langle\mathbf{x}, \mathbf{y}\rangle) .
$$

With this definition the reproducing kernel properties

$$
\forall \mathbf{y} \in \mathbb{S}^{d}: K^{(s)}(\langle\cdot, \mathbf{y}\rangle) \in H^{s}\left(\mathbb{S}^{d}\right) \text { and } \forall f \in H^{s}\left(\mathbb{S}^{d}\right) \forall \mathbf{x} \in \mathbb{S}^{d}: f(\mathbf{x})=\left\langle f, \mathrm{~K}^{(s)}(\langle\cdot, \mathbf{x}\rangle)\right\rangle_{H^{s}}
$$

can be immediately verified.

For $f \in H^{s}\left(\mathbb{S}^{d}\right), s>d / 2$, the integration error of the QMC method with node set $X_{N}$ is given by

$$
Q\left(X_{N}\right)(f):=\frac{1}{N} \sum_{\mathbf{x} \in X_{N}} f(\mathbf{x})-\int_{\mathbb{S}^{d}} f(\mathbf{x}) \mathrm{d} \sigma_{d}(\mathbf{x})=\left\langle f, R\left(X_{N}\right)\right\rangle_{H^{s}}
$$

where the function

$$
R\left(X_{N}\right)(\mathbf{y}):=\frac{1}{N} \sum_{\mathbf{x} \in X_{N}} \mathrm{~K}^{(s)}(\langle\mathbf{y}, \mathbf{x}\rangle)-1
$$

is called the representer of the integration error. It is now a consequence of elementary Hilbert space theory that the worst case error takes the form

$$
\text { wce }_{H^{s}}\left(X_{N}\right):=\sup _{\|f\|_{H^{s}}=1}\left|Q\left(X_{N}\right)(f)\right|=\left\|R\left(X_{N}\right)\right\|_{H^{s}} .
$$

The squared norm $\left\|R\left(X_{N}\right)\right\|^{2}=\left\langle R\left(X_{N}\right), R\left(X_{N}\right)\right\rangle$ can be expressed in terms of the kernel function $\mathrm{K}^{(s)}$ by means of

$$
\left\|R\left(X_{N}\right)\right\|_{H^{s}}^{2}=\frac{1}{N^{2}} \sum_{\mathbf{x}, \mathbf{y} \in X_{N}} \mathrm{~K}^{(s)}(\langle\mathbf{x}, \mathbf{y}\rangle)-1 .
$$

For a more precise explanation of this formalism we refer to [61]. The expression (2.33) is a special case of an energy functional as discussed further in Section 3 .

Taking a spherical $t$-design for the set $X_{N}$ in (2.33), the first $t$ terms in the LaplaceFourier expansion of $\mathrm{K}^{(s)}$ are annihilated:

$$
\text { wce }_{H^{s}}\left(X_{N}\right)^{2}=\sum_{\ell=t+1}^{\infty}(1+\ell)^{-2 s} Z(d, \ell) \frac{1}{N^{2}} \sum_{\mathbf{x}, \mathbf{y} \in X_{N}} P_{\ell}^{(d)}(\langle\mathbf{x}, \mathbf{y}\rangle) .
$$


Trivially estimating this sum and taking square roots would give a bound $\mathcal{O}\left(t^{d / 2-s}\right)$ for the worst case error.

A method is introduced in [128] and [129], which provides much better estimates for the worst case error in $H^{s}\left(\mathbb{S}^{2}\right)$. This method was extended and generalised to higher dimensions in [60]. This is now a standard technique in this context. While too technical to give a precise description here, we explain the two main steps. For a full description of the method we refer to [60].

First, the tail of the series defining the kernel

$$
\mathrm{K}_{t}^{(s)}(x):=\sum_{\ell=t+1}^{\infty}(1+\ell)^{-2 s} Z(d, \ell) P_{\ell}^{(d)}(x)
$$

is rewritten in terms of Jacobi polynomials instead of Legendre polynomials. This is done using the fact that the Legendre polynomials are special cases of Jacobi polynomials and that there exist connection formulas involving hypergeometric expressions (see [149]). After this transformation, the function $K_{t}^{(s)}$ is expressed as a polynomial of degree $t$ plus a series $\widetilde{K}_{t}^{(s)}$ involving higher order polynomials ("kernel splitting method"). The polynomial part is integrated exactly by the QMC method supported in $X_{N}$; the remaining part

$$
\sum_{\mathbf{x}, \mathbf{y} \in X_{N}} \widetilde{\mathrm{K}}_{t}^{(s)}(\langle\mathbf{x}, \mathbf{y}\rangle)
$$

is estimated using bounds for Jacobi polynomials. This part is rather delicate, since different estimates have to be used for $\langle\mathbf{x}, \mathbf{y}\rangle \in[-1+c / t, 1-c / t]$ and $|\langle\mathbf{x}, \mathbf{y}\rangle|>1-c / t$. Here, a coarse equidistribution property of spherical $t$-designs is used to obtain optimal order estimates: there exist constants $c_{1}$ and $c_{2}$, independent of $t$ and $N$, such that

$$
\#\left(X_{N} \cap C\left(\mathbf{x}, \frac{c_{1}}{t}\right)\right) \leq c_{2} N \sigma_{d}\left(C\left(\mathbf{x}, \frac{c_{1}}{t}\right)\right) .
$$

It was proved in [168] (see also [169]) that this is a general property of positive weight quadrature formulas, which integrate polynomials of degree $\leq t$ exactly; thus (2.35) holds a forteriori for spherical $t$-designs.

Using the technique described above, it was proved in [60] that the worst case integration error on $H^{s}\left(\mathbb{S}^{d}\right)$ (for $s>d / 2$ ) is

$$
\operatorname{wce}_{H^{s}}\left(X_{N}\right)=\mathcal{O}_{s, d}\left(t^{-s}\right) .
$$

Using the recent progress on the existence of spherical designs with optimal growth order (see [29]), this gives

$$
\text { wce }_{H^{s}}\left(X_{N}\right)=\mathcal{O}_{s, d}\left(N^{-s / d}\right)
$$

if $X_{N}$ is a spherical $t$-design with $N=\mathcal{O}\left(t^{d}\right)$. This is known to be the optimal order for the worst case error in $H^{s}\left(\mathbb{S}^{d}\right)$ (see [126, 127]).

The estimate (2.37) led to the following definition in [61]. Let $s>d / 2$, then a sequence of point sets $\left(X_{N}\right)$ on $\mathbb{S}^{d}$ is called a sequence of $Q M C$-designs for $H^{s}\left(\mathbb{S}^{d}\right)$, if (2.37) holds. 
The supremum over all $s$ for which (2.37) holds, is called the strength of the sequence $\left(X_{N}\right)$. By the above description, a sequence of spherical $t$-designs of optimal growth order $\mathcal{O}\left(t^{d}\right)$ has strength $\infty$. For a more detailed exposition of QMC-designs and their properties, we refer to the original paper [61]. These investigations were extended to the $L^{p}$-setting in [51].

\subsection{Applications in approximation and interpolation}

Well distributed point sets can also be used as sample points for interpolation formulas. Let $f: \mathbb{S}^{d} \rightarrow \mathbb{R}$ be a continuous function and $X_{N}$ a set of points with $N \geq Z(d+1, t)$. Observe that

$$
Z(d+1, t)=\sum_{k=0}^{t} Z(d, k)
$$

is the dimension of the space of all polynomials of total degree $\leq t$ on $\mathbb{S}^{d}$. Then we seek a polynomial $p$ of degree $\leq t$, such that

$$
p\left(\mathbf{x}_{i}\right)=f\left(\mathbf{x}_{i}\right) \quad \text { holds for } i=1, \ldots, N .
$$

Choosing $Y_{\ell, k}(\ell=0, \ldots, t, k=1, \ldots, Z(d, \ell))$ as a basis for the polynomials and $N=$ $Z(d+1, t)$, we have to solve a system of $N$ linear equations; the points must be chosen so that this system has full rank. One possible method for choosing the set $X_{N}$ is to maximise the modulus of the determinant of the system, which optimises the numerical stability of solving the system. This approach is in the spirit of finding the extremal value of a functional depending on the points, such as the energy functionals discussed in Section 3, This has been done in [184] for $d=2$.

In [6] it is proposed to use spherical $t$-designs as interpolation points; for $N \geq Z(d+1, t)$ the determinant of the matrix

$$
H_{t}:=\left(\sum_{j=1}^{N} Y_{\ell, k}\left(\mathbf{x}_{j}\right) Y_{\ell^{\prime}, k^{\prime}}\left(\mathbf{x}_{j}\right)\right)_{(\ell, k),\left(\ell^{\prime}, k^{\prime}\right)} 1 \leq \ell, \ell^{\prime} \leq t, \quad 1 \leq k \leq Z(d, \ell), \quad 1 \leq k^{\prime} \leq Z\left(d, \ell^{\prime}\right),
$$

is maximised under the constraint that the point set $X_{N}$ is a spherical $t$-design. This leads to the definition of well conditioned designs. In [6] this is worked out for $d=2$.

It should be mentioned that, based on the numerical experiments in [107, 123], there exist $t$-designs with $\left\lfloor(t+1)^{2} / 2\right\rfloor$ points for $t \leq 100$. In the context of interpolation, the number of points has to be chosen $\geq(t+1)^{2}$, which gives further freedom for choosing the points that can be used to maximise the determinant of $H_{t}$.

In [7], the application of spherical designs in the context of approximation of functions $f: \mathbb{S}^{d} \rightarrow \mathbb{R}$ is proposed. Usually, the approximation is computed by integrating $f$ against an approximation kernel $G_{L}$, which is a polynomial of degree $L$ in $\langle\mathbf{x}, \mathbf{y}\rangle$ (see [23, 170]). In [7] the integral is replaced by the equal weight quadrature rule given by a $2 L$-design $X_{N}$. This approach is worked out for $d=2$ and various classical approximation kernels. In order 
to make this procedure numerically more stable, a regularisation procedure is investigated: the polynomial $p$ is chosen to minimise

$$
\sum_{j=1}^{N}\left(p\left(\mathbf{x}_{j}\right)-f\left(\mathbf{x}_{j}\right)\right)^{2}+\lambda \sum_{j=1}^{N}\left(\mathcal{R} p\left(\mathbf{x}_{j}\right)\right)^{2}
$$

amongst all polynomials of degree $L$; here $\mathcal{R}$ is an operator on the space of polynomials and $\lambda>0$ is the regularisation parameter. The fact that $X_{N}$ is chosen as a $2 L$-design simplifies the linear algebra behind the least square approximation.

\section{Energy}

\subsection{Minimal energy in applications}

A surprising number of diverse applications can be formulated as a discrete or continuous minimal energy problem or a mixture of both. In the discrete setting this means finding a collection of $N$ distinct points in a subset $\Omega \subset \mathbb{R}^{p}, p \geq 1$, that minimises a discrete K-energy functional

$$
E_{\mathrm{K}, \mathrm{Q}}\left(\mathbf{x}_{1}, \ldots, \mathbf{x}_{N}\right):=\sum_{\substack{i=1 \\ i \neq j}}^{N} \sum_{j=1}^{N}\left[\mathrm{~K}\left(\mathbf{x}_{i}, \mathbf{x}_{j}\right)+\mathrm{Q}\left(\mathbf{x}_{i}\right)+\mathrm{Q}\left(\mathbf{x}_{j}\right)\right]
$$

among all sets of $N$ points from $\Omega$. The diagonal self-interaction terms are removed to allow singular kernels K. The external field Q is often taken to be zero. Evidently, further requirements on the set $\Omega$, the kernel $\mathrm{K}$, and the external field $\mathrm{Q}$ are needed to ensure existence of a solution. A suitable $\mathrm{Q}$ compatible with $\mathrm{K}$ introduces soft boundaries and thus prevents points from escaping to infinity in the case of unbounded sets $\Omega$. On the other hand, the restriction of the points to a finite set, fractal, torus or sphere of Hausdorff dimension $d \leq p$ introduces fractal or topological aspects. A standard assumption is that $\mathrm{K}$ is symmetric and lower semi-continuous on $\Omega \times \Omega$ and that $\mathrm{Q}$ is also lower semicontinuous on $\Omega$. This implies that the minimal K-energy problem with external field $\mathrm{Q}$ has a solution for every $N \geq 2$ when solved for an infinite compact set $\Omega$. A solution is called $N$-point minimal K-energy configuration associated with Q and its K-energy is equal to the minimum $N$-point $\mathrm{K}$-energy of $\Omega$ associated with $\mathrm{Q}$ given by

$$
\mathcal{E}_{\mathrm{K}, \mathrm{Q}}(\Omega ; N):=\inf \left\{E_{\mathrm{K}, \mathrm{Q}}\left(\mathbf{x}_{1}, \ldots, \mathbf{x}_{N}\right): \mathbf{x}_{1}, \ldots, \mathbf{x}_{N} \in \Omega\right\} .
$$

Furthermore, if $\mathcal{E}_{\mathrm{K}, \mathrm{Q}}(\Omega ; N)>0$ for all $N$, then the quantities $N(N-1) / \mathcal{E}_{\mathrm{K}, \mathrm{Q}}(\Omega ; N)$ form a non-increasing sequence that is bounded from below by lower semi-continuity 4 Thus the limit exists and it discriminates between two types of sets $\Omega$ depending on whether or not

\footnotetext{
${ }^{4}$ The reciprocals $\mathcal{E}_{\mathrm{K}, \mathrm{Q}}(\Omega ; N) /[N(N-1)]$ always form a non-decreasing sequence that may be unbounded.
} 
this limit vanishes. This gives rise to two different regimes characterised by a complete change in the nature of the minimization problem with regard to properties of the solution and methods that are used to study it. In the field-free setting $(\mathrm{Q} \equiv 0)$, the normalised discrete minimal $N$-point energy of $\Omega$, given by $\mathcal{E}_{\mathrm{K}, 0}(\Omega ; N) /[N(N-1)]$, is also known as the $N$ th diameter of $\Omega$. The limit in the extended sense (as $N \rightarrow \infty$ ) is called the transfinite diameter of $\Omega$.

A fundamental question concerns the "limit distribution" (if such exists) of a sequence $\left(X_{N}^{*}\right)$ of minimal energy configurations $X_{N}^{*}$ on $\Omega$ as $N \rightarrow \infty$; i.e., is there a (unique) Borel probability measure $\mu_{\Omega}^{*}$ supported on $\Omega$ that is the weak limit of the sequence formed by the discrete equal weight distribution supported on $X_{N}^{*}$,

$$
\mu_{X_{N}^{*}}:=\frac{1}{N} \sum_{j=1}^{N} \delta_{\mathbf{x}_{j}}
$$

Let $\mathcal{M}(\Omega)$ denote the collection of Borel probability measures supported on $\Omega$. The analogous continuous energy problem is to find a measure in $\mathcal{M}(\Omega)$ that minimises the weighted $\mathrm{K}$-energy associated with $\mathrm{Q}$ given by

$$
\mathcal{I}_{\mathrm{K}, \mathrm{Q}}(\mu):=\mathcal{I}_{\mathrm{K}}(\mu)+2 \int_{\Omega} \mathrm{Q}(\mathbf{x}) \mathrm{d} \mu(\mathbf{x})
$$

among all measures in $\mathcal{M}(\Omega)$, where

$$
\mathcal{I}_{\mathrm{K}}(\mu):=\int_{\Omega} \int_{\Omega} \mathrm{K}(\mathbf{x}, \mathbf{y}) \mathrm{d} \mu(\mathbf{x}) \mathrm{d} \mu(\mathbf{y})
$$

is the K-energy of $\mu \in \mathcal{M}(\Omega)$. A minimising measure $\mu_{\mathrm{K}, \mathrm{Q} ; \Omega} \in \mathcal{M}(\Omega)$ with

$$
\mathcal{I}_{\mathrm{K}, \mathrm{Q}}\left(\mu_{\mathrm{K}, \mathrm{Q} ; \Omega}\right)=W_{\mathrm{K}, \mathrm{Q}}(\Omega):=\inf \left\{\mathcal{I}_{\mathrm{K}, \mathrm{Q}}(\mu) \mid \mu \in \mathcal{M}(\Omega)\right\},
$$

is called a $\mathrm{K}$-extremal (or positive equilibrium) measure on $\Omega$ associated with $\mathrm{Q}$. In the field free setting $\mathrm{Q} \equiv 0$, a minimising measure $\mu_{K ; \Omega}$ is called $\mathrm{K}$-equilibrium measure on $\Omega$. In this case, the K-energy $\mathcal{I}_{\mathrm{K}}\left(\mu_{K ; \Omega}\right)$ is equal to the Wiener energy of $\Omega$,

$$
W_{\mathrm{K}}(\Omega):=\inf \left\{\mathcal{I}_{\mathrm{K}}(\mu) \mid \mu \in \mathcal{M}(\Omega)\right\} .
$$

A fundamental question concerns the relationship between the Wiener energy of $\Omega$, the transfinite diameter of $\Omega$, and the Chebyshev constant of $\Omega$ which is the limit as $N \rightarrow \infty$ of the $N$ th Chebyshev constant of $\Omega$ defined as

$$
M_{\mathrm{K}}(\Omega ; N):=\sup _{\mathbf{x}_{1}, \ldots, \mathbf{x}_{N} \in \Omega} \inf _{\mathbf{x} \in \Omega} \frac{1}{N} \sum_{j=1}^{N} \mathrm{~K}\left(\mathbf{x}, \mathbf{x}_{j}\right)
$$

see [90] and references cited therein for further details.

We conclude this section by a discussion of applications that make use of the minimal energy problem or are related to it. 
The Thomson problem and its generalizations. A classical problem in electrostatics is to find the distribution of $N$ unit point charges on a conductor in the most stable equilibrium (the charges interact according to the Coulomb potential $1 / r$, where $r$ is the Euclidean distance between two interacting charges). This leads to a minimization problem for the potential energy of the discrete charge system named after J. J. Thomson who posed it for the sphere [191].5 Generalizations of Thomson problem that utilise a Riesz $s$-potential $1 / r^{s}$ are used, e.g., to model multi-electron bubbles and arrangements of protein subunits which form the shells (capsids) of viruses; see [38] (also [40, 41]) for a discussion. In material physics (see 63]) minimal energy points on the sphere have been used to model "spacer particles" in powders which ensure that spherical host-particles do not touch.

Polarization. A problem related to finding the minimal K-energy configurations on a compact set $\Omega \subset \mathbb{R}^{p}$ is to find optimal $N$-point $K$-polarization configurations on $\Omega$, which are configurations on $\Omega$ that maximise the minimal value over $\Omega$ of the potential

$$
\frac{1}{N} \sum_{j=1}^{N} \mathrm{~K}\left(\mathbf{x}, \mathbf{x}_{j}\right) .
$$

An optimal configuration realises the $N$ th Chebyshev constant of $\Omega$. For a Riesz kernel $\mathrm{K}_{s}(\mathbf{x}, \mathbf{y})=1 /[\mathrm{m}(\mathbf{x}, \mathbf{y})]^{s}$ with metric $\mathrm{m}$ and $s>0$, one has the following duality: the minimal $s$-energy configurations tend to best-packing configurations on $\Omega$ as $s \rightarrow \infty$, whereas the optimal $s$-polarization configurations on $\Omega$ tend to best-covering configurations on $\Omega$. Questions concerning optimal polarization configurations, their limit distributions, polarization inequalities, asymptotic behaviour of the $N$ th Chebyshev constant are addressed in [5, 32, 89, 114, 166]. The paper [89] proposes a conjecture for the dual of the Poppy-seed Bagel theorem 6 for optimal polarization points (which is proven for the boundary case $s=\operatorname{dim}(\Omega)$ in [32]).

Smale's 7th Problem. In the seminal work [183], M. Shub and S. Smale define a condition number of a polynomial at a point in $\mathbb{C}$, which connects the problem of solving a polynomial equation with the discrete minimal logarithmic energy problem on $\mathbb{S}^{2}$ (see Section 3.2). They show that a monic polynomial whose zeros are the stereographic projection of minimal logarithmic energy points on the Riemann sphere, called "elliptic Fekete polynomial", is "well conditioned" and thus gives a good starting polynomial for a homotopy method for solving a polynomial equation or system (see [182]). This is the background for Smale's 7th problem [186]: Find an algorithm which, on input $N$, outputs distinct points $\mathbf{x}_{1}, \ldots, \mathbf{x}_{N}$ on the Riemann sphere such

\footnotetext{
${ }^{5}$ Recently, T. LaFave Jr. [138] investigated correspondences between the Thomson problem and atomic electronic structure, and he applied discrete transformations to the Thomson problem in [139] to study the minimal Coulomb energy.

${ }^{6} \mathrm{Cf}$. http://news.vanderbilt.edu/2004/11/the-poppy-seed-bagel-theorem-59497/
} 
that

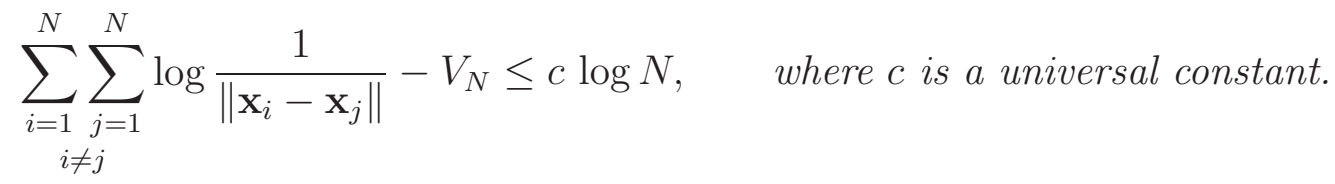

Here $V_{N}$ is the minimal logarithmic energy of $N$ points on the Riemann sphere. Smale further specifies that the algorithm is a real number algorithm in the sense of Blum, Cucker, Shub, and Smale (see [27]) with halting time polynomial in $N$. In [19], C. Beltrán showed that there are $N$-point sets with logarithmic energy that differs from $V_{N}$ by at most $1 / 9$. These points are rational with coordinates of order $\log _{2} N$ bit length. Thus, there exists an exponential running time algorithm. Mean value considerations yield that the typical logarithmic energy of $N$ i.i.d. uniformly distributed random points on the Riemann sphere is of the form $\frac{1}{2} N^{2}-\frac{1}{2} N$. One the other hand, D. Armentano, C. Beltrán, and M. Shub [9] 7 observe that the typical logarithmic energy of the zeros of certain random polynomials, $\frac{1}{2} N^{2}-\frac{1}{2} N \log N-\frac{1}{2} N$, is surprisingly small in the sense that the first two terms in the asymptotics of the minimal $N$-point logarithmic energy for the Riemann sphere are recovered. A recent account of the state of the art regarding Smale's 7th problem can be found in [20] (see also [21]).

Log gases, Coulomb gases, and random matrices. A log or Coulomb gas is a system of interacting particles in which the repelling interaction is governed by a logarithmic or Coulomb potential. An external field confines the particles to a finite volume of the space. Typically, the mean-field regime is considered. In this setting the number $N$ of particles is large and the pair-interaction strength (coupling parameter) scales as the inverse of $N$. The Hamiltonian

$$
H\left(\mathbf{x}_{1}, \ldots, \mathbf{x}_{N}\right):=\sum_{\substack{i=1 \\ i \neq j}}^{N} \sum_{j=1}^{N} k\left(\mathbf{x}_{i}-\mathbf{x}_{j}\right)+N \sum_{i=1}^{N} V\left(\mathbf{x}_{i}\right), \quad \mathbf{x}_{1}, \ldots, \mathbf{x}_{N} \in \mathbb{R}^{d},
$$

where $k(\mathbf{x})=-\log \|\mathbf{x}\|$ if $d=2$ or $k(\mathbf{x})=\|\mathbf{x}\|^{2-d}$ if $d \geq 3$, is minimised 8 Very recent progress provides a deep connection with the discrete minimum energy problem on the sphere (see discussion at the end of Section 3.6 ). We refer further to S. R. Nodari and S. Serfaty [161] and N. Rougerie and S. Serfaty [171]. The concept of renormalised energy can be also successfully applied to random matrices; see A. Borodin and S. Serfaty [37]. For the connection between log gases and random matrix applications and theory we refer to the book of P. Forrester [95] and, e.g., T. Claeys,

\footnotetext{
${ }^{7}$ The paper [94] gives a generalization to higher dimensions and other manifolds.

${ }^{8}$ Instead of the harmonic potentials one can also consider Riesz $s$-potentials $\|\cdot\|^{-s}$. For $s \geq d$ one needs to adjust the coupling parameter to ensure comparability between the pair-interaction part and external field part.
} 
A. B. J. Kuijlaars and M. Vanlessen [71] and A. Mays [152]. A related problem is the discrete energy of periodic point sets in the Euclidean space; see D. P. Hardin, E. B. Saff, and B. Simanek [117].

Half-toning. Loosely speaking, half-toning is a way of creating an illusion of a grey-value image by appropriately distributing black dots. In [108], M. Gräf, D. Potts, and G. Steidl show how the process of half-toning can be seen as a numerical integration process with the aim to minimise a worst-case error which can be interpreted as an external field problem where the picture drives the external field which guides the interacting points; see also [190] and [105].

Maximizing Determinants. Points that maximise a Vandermonde-like determinant are well-suited for interpolation and numerical integration. They are called Fekete points due to the paper [93] by M. Fekete. Given a compact set in the complex plane, Fekete points are, indeed, minimal logarithmic energy points. However, in higher dimensions, Fekete's optimisation problem is different from minimising the logarithmic energy. I. H. Sloan and R. S. Womersley [184] used the logarithm of the determinant of an interpolation matrix to calculate extremal systems which yield interpolatory cubature rules with positive weights on the sphere. J. Marzo and J. Ortega-Cerdá [151] established that Fekete (or extremal) points are asymptotically uniformly distributed.

Diffusion on a sphere with localised traps. As an application in cellular signal transport, D. Coombs, R. Straube, and M. Ward [79] calculate the principal eigenvalue for the Laplacian on the unit sphere in the presence of $N$ traps on the surface of the sphere of asymptotically small radii. The positions of the traps are chosen to minimise the discrete logarithmic energy given in (3.3) below.

\subsection{The discrete and continuous minimal logarithmic and Riesz energy problem}

The discrete logarithmic energy problem on $\mathbb{S}^{d}$ is concerned with the properties of $N$ point configurations $\left\{\mathbf{x}_{1}^{*}, \ldots, \mathbf{x}_{N}^{*}\right\} \subset \mathbb{S}^{d}$ that maximise the product of all mutual pairwise Euclidean distances

$$
\prod_{\substack{i=1 \\ i \neq j}}^{N} \prod_{\substack{j=1 \\ N}}^{N} \mathbf{x}_{i}-\mathbf{x}_{j} \|
$$

or equivalently, minimise the discrete logarithmic energy

$$
E_{\log }\left(\mathbf{x}_{1}, \ldots, \mathbf{x}_{N}\right):=\sum_{i=1}^{N} \sum_{\substack{j=1 \\ i \neq j}}^{N} \log \frac{1}{\left\|\mathbf{x}_{i}-\mathbf{x}_{j}\right\|}
$$

over all $N$-point configurations $\left\{\mathbf{x}_{1}, \ldots, \mathbf{x}_{N}\right\}$ on $\mathbb{S}^{d}$. The discrete logarithmic energy can be understood as a limiting case (as $s \rightarrow 0$ ) of the Riesz $s$-energy

$$
E_{s}\left(\mathbf{x}_{1}, \ldots, \mathbf{x}_{N}\right):=\sum_{i=1}^{N} \sum_{\substack{j=1 \\ i \neq j}}^{N} \frac{1}{\left\|\mathbf{x}_{i}-\mathbf{x}_{j}\right\|^{s}}
$$


i.e., $E_{s}\left(\mathbf{x}_{1}, \ldots, \mathbf{x}_{N}\right)=N(N-1)+s E_{\log }\left(\mathbf{x}_{1}, \ldots, \mathbf{x}_{N}\right)+o(s)$ as $s \rightarrow 0$.

The discrete Riesz s-energy problem for $s>0$ is concerned with the properties of $N$ point configurations $\left\{\mathbf{x}_{1}^{*}, \ldots, \mathbf{x}_{N}^{*}\right\} \subset \mathbb{S}^{d}$ that minimise the Riesz $s$-energy over all $N$-point configurations $\left\{\mathbf{x}_{1}, \ldots, \mathbf{x}_{N}\right\}$ on $\mathbb{S}^{d}$. For convenience we set $\mathrm{k}_{s}(\mathbf{x}, \mathbf{y}):=-\log \|\mathbf{x}-\mathbf{y}\|$ for $s=\log$ and $\mathrm{k}_{s}(\mathbf{x}, \mathbf{y}):=1 /\|\mathbf{x}-\mathbf{y}\|^{s}$ for $s \in \mathbb{R}$. Then we are interested in the optimal $N$-point s-energy of an infinite compact set $\Omega \subset \mathbb{S}^{d}$ defined by

$$
\mathcal{E}_{s}(\Omega ; N):= \begin{cases}\min \left\{E_{s}\left(\mathbf{x}_{1}, \ldots, \mathbf{x}_{N}\right) \mid \mathbf{x}_{1}, \ldots, \mathbf{x}_{N} \in \Omega\right\} & \text { for } s=\log \text { or } s \geq 0, \\ \max \left\{E_{s}\left(\mathbf{x}_{1}, \ldots, \mathbf{x}_{N}\right) \mid \mathbf{x}_{1}, \ldots, \mathbf{x}_{N} \in \Omega\right\} & \text { for } s<0 .\end{cases}
$$

Observe that $\mathcal{E}_{0}(\Omega ; N)=N^{2}-N$ (which is attained by any $N$-point set on $\Omega$ ). The Riesz $s$-kernel is conditionally positive definite of order 1 for $-2<s<0$. Alternatively, as in the setting of numerical integration on $\mathbb{S}^{d}$, one can minimise

$$
2 \int_{\Omega} \int_{\Omega}\|\mathbf{x}-\mathbf{y}\|^{-s} \mathrm{~d} \sigma_{d}(\mathbf{x}) \mathrm{d} \sigma_{d}(\mathbf{y})-\sum_{i=1}^{N} \sum_{j=1}^{N}\left\|\mathbf{x}_{i}-\mathbf{x}_{j}\right\|^{-s}
$$

in this case. The papers [115, 173] are standard references for the discrete logarithmic and Riesz s-energy problem.

The s-potential and the s-energy of a measure $\mu$ in the class $\mathcal{M}(\Omega)$ of Borel probability measures supported on $\Omega$ are given, respectively, by

$$
U_{s}^{\mu}(\mathbf{x}):=\int_{\Omega} \mathrm{k}_{s}(\mathbf{x}, \mathbf{y}) \mathrm{d} \mu(\mathbf{y}), \quad \mathbf{x} \in \mathbb{R}^{d+1}, \quad \mathcal{I}_{s}(\mu):=\int_{\Omega} \int_{\Omega} \mathrm{k}_{s}(\mathbf{x}, \mathbf{y}) \mathrm{d} \mu(\mathbf{x}) \mathrm{d} \mu(\mathbf{y}) .
$$

For $s>0$, the s-capacity of $\Omega$ is the reciprocal of the Wiener energy $\inf \left\{\mathcal{I}_{s}(\mu) \mid \mu \in \mathcal{M}(\Omega)\right\}$. When the Wiener energy is finite, it will be denoted by $W_{s}(\Omega)$. Because of the possibility of negative logarithmic energy integral, we set $\operatorname{cap}_{\log }(\Omega):=\exp \left(-\inf \left\{\mathcal{I}_{\log }(\mu) \mid \mu \in \mathcal{M}(\Omega)\right\}\right)$. When finite, the infimum is denoted by $W_{\log }(\Omega)$. The lower semi-continuous logarithmic kernel is bounded from below and thus the kernel $\mathrm{k}_{s}$ (plus a constant) is strictly positive definite for $s=\log$ and $0<s<d$. Consequently, the $s$-equilibrium measure $\mu_{\Omega, s}$ on $\Omega$ is unique for every compact set $\Omega \subset \mathbb{R}^{p}$ with finite $s$-energy; see [36, 140]. For the range $-2<s<0$, one also has a unique $s$-equilibrium measure on $\Omega$; see [26] for the potential theoretic quantities and variational inequalities.

In the remaining section we consider the sphere $\mathbb{S}^{d}$ and subsets of $\mathbb{S}^{d}$. An external field is a lower semi-continuous function $\mathrm{Q}: \mathbb{S}^{d} \rightarrow(-\infty, \infty]$ such that $\mathrm{Q}(\mathrm{x})<\infty$ on a set of positive Lebesgue surface measure. We note that the lower semi-continuity implies the existence of a finite $c_{\mathrm{Q}}$ such that $\mathrm{Q}(\mathrm{x}) \geq c_{\mathrm{Q}}$ for all $\mathbf{x} \in \mathbb{S}^{d}$. The weighted energy associated with $\mathrm{Q}(\mathbf{x})$ is then given by

$$
\mathcal{I}_{\mathrm{Q}, s}(\mu):=\mathcal{I}_{s}(\mu)+2 \int \mathrm{Q}(\mathbf{x}) \mathrm{d} \mu(\mathbf{x}), \quad \mu \in \mathcal{M}\left(\mathbb{S}^{d}\right) .
$$

We recall from [87] the following Frostman-type result that deals with existence and uniqueness of the $s$-extremal measure on $\Omega$ associated with Q and its characterization in terms of 
weighted potentials. The result is stated for the Riesz case but a similar result holds also for the logarithmic case. The potential theory used in the context of this survey is formulated by G. Björck 26] (dealing with Riesz potential with negative exponent), by E. B. Saff, and V. Totik 174] (logarithmic external field problem in the plane), by N. S. Landkof [140] (Riesz and logarithmic potential and general reference) and, in particular, for Riesz external field problems by N. V. Zoriu [200, 201].

Proposition 3.1. Let $0<s<d$. For the minimal energy problem on $\mathbb{S}^{d}$ with external field $\mathrm{Q}$ the following properties hold:

(a) $W_{\mathrm{Q}, s}:=\inf \left\{\mathcal{I}_{\mathrm{Q}, s}(\mu) \mid \mu \in \mathcal{M}\left(\mathbb{S}^{d}\right)\right\}$ is finite.

(b) There is a unique s-extremal measure $\mu_{\mathrm{Q}, s} \in \mathcal{M}\left(\mathbb{S}^{d}\right)$ associated with $\mathrm{Q}$. Moreover, the support $S_{\mathrm{Q}, s}:=\operatorname{supp}\left(\mu_{\mathrm{Q}, s}\right)$ of this measure is contained in the compact set $E_{M}:=\{\mathbf{x} \in$ $\left.\mathbb{S}^{d}: \mathrm{Q}(\mathbf{x}) \leq M\right\}$ for some $M>0$.

(c) The measure $\mu_{\mathrm{Q}, s}$ satisfies the variational inequalities

$$
\begin{array}{ll}
U_{s}^{\mu_{\mathrm{Q}, s}}(\mathbf{x})+\mathrm{Q}(\mathbf{x}) \geq F_{\mathrm{Q}, s} & \text { q.e. on } \mathbb{S}^{d}, \\
U_{s}^{\mu_{\mathrm{Q}, s}}(\mathbf{x})+\mathrm{Q}(\mathbf{x}) \leq F_{\mathrm{Q}, s} & \text { everywhere on } S_{\mathrm{Q}, s},
\end{array}
$$

where

$$
F_{\mathrm{Q}, s}:=W_{\mathrm{Q}, s}-\int \mathrm{Q}(\mathbf{x}) \mathrm{d} \mu_{\mathrm{Q}, s}(\mathbf{x})
$$

(d) Inequalities (3.6) and (3.7) completely characterise the s-extremal measure $\mu_{\mathrm{Q}}$ in the sense that if $\nu \in \mathcal{M}\left(\mathbb{S}^{d}\right)$ is a measure with finite s-energy such that for some constant $C$ we have

$$
\begin{array}{ll}
U_{s}^{\nu}(\mathbf{x})+\mathrm{Q}(\mathbf{x}) \geq C & \text { q.e. on } \mathbb{S}^{d} \\
U_{s}^{\nu}(\mathbf{x})+\mathrm{Q}(\mathbf{x}) \leq C & \text { everywhere on } \operatorname{supp}(\nu)
\end{array}
$$

then $\nu=\mu_{\mathrm{Q}, s}$ and $C=F_{\mathrm{Q}, s}$.

A property holds quasi-everywhere if the exceptional set has s-capacity zero. If $\mathrm{Q}$ is continuous on $\mathbb{S}^{d}$, then the inequalities (3.6) and (3.9) hold everywhere on $\mathbb{S}^{d}$.

In principle, once $\operatorname{supp}\left(\mu_{\mathrm{Q}}\right)$ is known, then the measure $\mu_{\mathrm{Q}}$ can be recovered by solving an integral equation for the weighted $s$-potential arising from (3.9) and (3.10). Finding $\operatorname{supp}\left(\mu_{\mathrm{Q}}\right)$ when it is a proper subset of $\mathbb{S}^{d}$ can be a very difficult problem. It is a substantially easier task to find a signed measure that has constant weighted $s$-potential everywhere on $\mathbb{S}^{d}$. Given a compact subset $\Omega \subset \mathbb{S}^{d}$ with $\operatorname{cap}_{s}(\Omega)>0$ and an external field Q, there is a unique (if it exists, see [52, Lemma 23]) signed $s$-equilibrium measure $\eta_{\mathrm{K}, \mathrm{Q}}$ supported on $\Omega$ of total charge one associated with $\mathrm{Q}$ with constant weighted s-potential; i.e.,

$$
U_{s}^{\eta_{\mathrm{K}, \mathrm{Q}}}(\mathbf{x})+\mathrm{Q}(\mathbf{x})=G_{\mathrm{K}, \mathrm{Q}, s} \quad \text { everywhere on } \Omega
$$


for some constant $G_{\mathrm{K}, \mathrm{Q}, s}$. A remarkable connection exists to the analogue of the MhaskarSaff functional from classical planar potential theory ([154] and [174, Chapter IV, p. 194]) given by

$$
\mathcal{F}_{s}\left(\Omega^{\prime}\right):=W_{s}\left(\Omega^{\prime}\right)+\int \mathrm{Q}(\mathbf{x}) \mathrm{d} \mu_{\Omega^{\prime}}(\mathbf{x}), \quad \Omega^{\prime} \subset \mathbb{S}^{d} \text { compact with cap } \operatorname{cop}_{s}\left(\Omega^{\prime}\right)>0
$$

where $W_{s}\left(\Omega^{\prime}\right)$ is the $s$-energy of $\Omega^{\prime}$ and $\mu_{\Omega^{\prime}}$ is the $s$-equilibrium measure (without external field) on $\Omega^{\prime}$. Namely, if the signed $s$-equilibrium on a compact set $\Omega^{\prime}$ associated with Q exists, then $\mathcal{F}_{s}\left(\Omega^{\prime}\right)=G_{\mathrm{K}, \mathrm{Q}, s}$. The essential property of the $\mathcal{F}_{s^{-}}$-functional is that it is minimised for the support of the $s$-extremal measure (see [53, Proposition 8] for a precise statement). The papers [52, 53, 54, 87] determine the signed $s$-equilibrium on the full sphere and on spherical caps associated with logarithmic and Riesz s-external fields due to a single point charge (or a axis-supported superposition of such fields) to derive separation results for minimal Riesz $s$-energy points and to characterise the $s$-extremal measure on $\mathbb{S}^{d}$. The use of balayage techniques (the signed equilibrium can be expressed as the difference of two balayage measures) together with a restricted principle of domination and a restricted maximum principle yields that the Riesz parameter $s$ is restricted to the interval $[d-2, d)$. New phenomena occur when $s=d-2$. For example, a boundary charge appears in the signed $(d-2)$-equilibrium for a spherical cap which vanishes when the cap coincides with the support of the $s$-extremal measure on $\mathbb{S}^{d}$. Numerical methods for external field problems for Riesz potentials have been developed and applied in [111, 112, 113, 163].

\subsection{The distribution of minimal logarithmic and Riesz energy points}

Let $\Omega$ be an infinite compact set $\Omega \subset \mathbb{R}^{p}$ with Hausdorff dimension $d$ having finite logarithmic or Riesz $s$-energy $(s>0)$ and $\mathrm{Q} \equiv 0$. Then classical potential theory implies that the minimal energy configurations $X_{N}^{*}$ on $\Omega$ are distributed according to the unique equilibrium measure $\mu_{\Omega}$ on $\Omega$ and the discrete measures $\mu_{X_{N}^{*}}$ have $\mu_{\Omega}$ as a weak limit. The $s$-equilibrium measure on $\mathbb{S}^{d}$ is the uniform measure $\sigma_{d}$. In general, the measure $\mu_{\Omega}$ will not be uniform as the example of a circular torus shows (see [118] and [55, 56]).9 In this potential theoretic regime $(s=\log$ or $0<s<d)$, the support of $\mu_{\Omega}$ depends on whether the kernel is superharmonic, harmonic or subharmonic (see [140]). In the superharmonic case $(s=\log$ or $0<s<p-2)$, the measure $\mu_{\Omega}$ is supported on the outer boundary (i.e., the boundary of $\Omega$ shared with the unbounded component of the ambient space $\mathbb{R}^{p}$ ), whereas in the strictly subharmonic case, the measure $\mu_{\Omega}$ can be supported on all of $\Omega$.

The intuition is that in the regime $s=\log$ or $0<s<d$ global effects dominate (points interact as if they are subject to long-range forces and the range increases as $s$ becomes smaller). In the hypersingular case $s>d$, local effects dominate (points interact as if they are responding to a short range force). Both kinds of interactions intermingle when $s=d$.

In the hypersingular case $s \geq d$, the energy integral attains $+\infty$ for every $\mu \in \mathcal{M}(\Omega)$. Geometric measure theory yields that the limiting distribution of a sequence $\left(X_{N}^{*}\right)$ of

\footnotetext{
${ }^{9}$ It seems to be unresolved for which $\Omega$ the $s$-equilibrium measure on $\Omega$ is the uniform measure on $\Omega$.
} 
minimal Riesz $s$-energy $N$-point sets on $\Omega$ (even asymptotically $s$-energy minimising would suffice) is uniformly distributed with respect to the $d$-dimensional Hausdorff measure $\mathcal{H}_{d}$,

$$
\mu_{X_{N}^{*}} \stackrel{*}{\rightarrow} \frac{\left.\mathcal{H}_{d}\right|_{\Omega}}{\mathcal{H}_{d}(\Omega)} \quad \text { as } N \rightarrow \infty,
$$

for a large class of sets $\Omega$ with $\mathcal{H}_{d}(\Omega)>0$ (see [34] and earlier work [33, 115, 116]). It should be noted that in the case $s=d$ an additional regularity assumption on $\Omega$ is required. In the limit $s \rightarrow \infty$ only the nearest-neighbour interaction matters and the optimal solutions are best-packing configurations which solve the Tammes problem [189]. The paper [34] also shows analogous results for weighted Riesz $s$-energy

$$
\sum_{\substack{i=1 \\ i \neq j}}^{N} \sum_{\substack{j=1 \\ N}}^{N} \frac{w\left(\mathbf{x}_{i}, \mathbf{x}_{j}\right)}{\left\|\mathbf{x}_{i}-\mathbf{x}_{j}\right\|^{s}}
$$

where $w$ is (almost everywhere) continuous and positive on the diagonal (called CPD weight function). We remark that the case $s=d$ for $\mathbb{S}^{d}$ has already been dealt with in [100] using results from [136]. Furthermore, in [66] it was shown that the s-equilibrium measures on $\Omega$ converge to the normalised $d$-dimensional Hausdorff measure restricted to $\Omega$ as $s \rightarrow d^{-}$under rather general assumptions on $\Omega$. We remark that I. Pritzker [165] studied the discrete approximation of the equilibrium measure on a compact set $\Omega \subset \mathbb{R}^{p}$, $p \geq 2$, with positive $s$-capacity by means of points which do not need to lie inside $\Omega$. He also obtained discrepancy estimates in the harmonic case. The properties of so-called greedy K-energy points were studied by A. López García and E. B. Saff [145].

Summarizing, for $-2<s<0, s=\log$ and $s>0$, the (asymptotically) $s$-energy minimising $N$-point configuration on $\mathbb{S}^{d}$ are uniformly distributed with respect to the surface area measure $\sigma_{d}$.

In certain applications one prefers to generate well distributed $N$-point sets on a compact $d$-rectifiable set in $\mathbb{R}^{p}$ which have a prescribed non-uniform asymptotic distribution $\rho$ with respect to $\mathcal{H}_{d}$ as $N \rightarrow \infty$. It is shown in [119] that such points can be obtained by minimising the energy of $N$ points on $\Omega$ interacting via a weighted power law potential $w(\mathbf{x}, \mathbf{y}) /\|\mathbf{x}-\mathbf{y}\|^{s}$, where $s>d$ and $w(\mathbf{x}, \mathbf{y}):=[\rho(\mathbf{x}) \rho(\mathbf{y})]^{-s /(2 d)}$. Furthermore, such point sets are "quasi-uniform" in the sense that the ratio of the covering radius to the separation distance is uniformly bounded in $N$. As mentioned in the introduction, quasi-uniformity is crucial for a number of numerical methods (see [96, 141, 177]). S. V. Borodachov, D. P. Hardin, and E. B. Saff show in 35] that it suffices to use a varying truncated weight $w(\mathbf{x}, \mathbf{y}) \Phi\left(\|\mathbf{x}-\mathbf{y}\| / r_{N}\right)$. Thus only those pairs of points that are located at a distance of at most $r_{N}=C_{N} N^{-1 / d}$ from each other contribute to the energy sum. (The positive sequence $\left(C_{N}\right)$ can be taken to tend to $\infty$ as slowly as one wishes.) In this way, under suitable assumptions, the complexity of the energy computation can be greatly reduced, leading to order $N C_{N}^{d}$ computations for generating "low energy" $N$-point approximations 10

${ }^{10}$ We note that in the astronomical community a hierarchical equal area iso-latitude pixelisation 
A point charge approaching the sphere subject to the same law of interaction as the points on the sphere affects the charge distribution on the sphere. Sufficiently close to the sphere, it will generate a spherical cap with zero charge. The papers [52, 53, 54], in particular, provide explicit representations of the charge distributions due to a single external charge. They also address a question attributed to A. A. Gonchar, namely to find a critical distance from the sphere surface of a point charge generating the external field so that the support of the $s$-extremal measure on $\mathbb{S}^{d}$ only just becomes the whole sphere. In the harmonic case this distance is characterised by the largest zero of polynomials dubbed Gonchar polynomials 11

For a small $N$, numerical optimization methods can be used to find putative minimal Riesz $s$-energy configurations. T. Erber and G. M. Hockney [88] noticed that the number of local minimal energy configurations (most of which are not global ones) seems to grow exponentially with $N$. Most of the numerical data is for the 2-sphere and for the Coulomb case and a few other values of $s$. Regarding data, we refer to online resources [39, 124, 198] and a more recent study of the energy landscape in [64, 65], whereas [22] provides a complexity analysis for the logarithmic case. Higher dimensional configurations have also been investigated (see [13]). In [160] monotonicity properties of the second discrete derivative were considered and led to new putative low-energy configurations in two cases. Numerical results in [153] suggest that a minimal $s$-energy $N$-point set may transit through one or more (depending on $N$ ) basic configuration as $s$ grows 12 The smallest only partly resolved problem is the five point problem on $\mathbb{S}^{2}$. Five points cannot form a universally optimal system [74] 13 Melnyk et al. [153] identified two basic configurations: triangular bi-pyramid and quadratic pyramid. According to numerical results, the regular triangular bi-pyramid is the putative energy-minimising configuration for $2 \leq s \leq 15.048077392 \ldots$, whereas for higher values of $s$ it seems to be the square pyramid (with adjusted height); see also [160] for a finer analysis. Moreover, it is shown in [28] that there are sequences of $s$-energy minimising configurations that tend to a square pyramid best packing configuration as $s \rightarrow \infty$. In general, the five point problem is a difficult problem to analyse rigorously. Recently, the papers [179] (for the Coulomb case $s=1$ and for $s=2$ ) and [131] (for sum of distances, $s=-1$ ) provided computer-assisted proofs that the triangular bi-pyramid is optimal, whereas in the logarithmic case a conventional proof was given in [86]. In [194] a bi-quadratic energy functional is considered. Other rigorously proved minimising configurations are rare and are often universally optimal. Proved minimising configurations on $\mathbb{S}^{2}$

(HEALPix [97]) is used to generate large numbers of uniformly distributed points on $\mathbb{S}^{2}$; cf. also 142]. A. Holhos and D. Roşca [130] study Riesz energy of points derived from an area-preserving map from the 2-sphere to the octahedron.

${ }^{11}$ For $d=2$ and $d=4$, the critical distance is the golden ratio and the plastic number.

${ }^{12}$ The phenomenon of transiting through several basic types of configurations can also be observed in the external field setting when $s$ is fixed but the distance of the external field source varies (see [53, Figure 4]).

${ }^{13} \mathrm{~A}$ universally optimal point set minimises all energy functionals with kernels of the form $\mathrm{K}(\mathbf{x}, \mathbf{y})=$ $f\left(\|\mathbf{x}-\mathbf{y}\|^{2}\right)$, where $f$ is a completely monotonic $C^{\infty}$ function like the Riesz potential $1 / r^{s}$ for $s>0$, meaning that $(-1)^{k} f^{(k)}(x) \geq 0$ for all $k$; see [74]. 
are the antipodal and equilateral configuration and the Platonic solids with $N=4,6$ and 12 vertices. For higher dimensions, we refer to [74, Table 1], [11] and [77, Section 5.3].

\subsection{Asymptotic expansion of minimal Riesz energy}

Let $s>0$. As sets we shall consider the unit sphere $\mathbb{S}^{d}$ and, more generally, infinite compact sets $\Omega \subset \mathbb{R}^{p}$ with Hausdorff dimension $0<d \leq p$. The leading term of the asymptotic expansion of the $N$-point Riesz $s$-energy of $\Omega$ is well-understood if $\Omega$ has positive $s$-capacity (i.e., finite Riesz s-energy). This is the potential-theoretic regime. A standard argument from classical potential theory yields that the positive quantities $N(N-1) / \mathcal{E}_{s}(\Omega ; N)$ form a monotonically decreasing sequence. The $\operatorname{limit}_{\operatorname{diam}_{s}}(\Omega)$, called the generalised transfinite diameter of $\Omega$, is equal to the $s$-capacity of $\Omega$ (cf. [164]). Thus, the leading term of $\mathcal{E}_{s}(\Omega ; N)$ grows like $N^{2}$ as $N \rightarrow \infty$ and the leading coefficient is given by the Riesz $s$-energy of $\Omega$, or equivalently, by the reciprocals of the $s$-capacity and transfinite diameter of $\Omega$ :

$$
\lim _{N \rightarrow \infty} \frac{\mathcal{E}_{s}(\Omega ; N)}{N^{2}}=W_{s}(\Omega)=\frac{1}{\operatorname{cap}_{s}(\Omega)}=\frac{1}{\operatorname{diam}_{s}(\Omega)} .
$$

For $0<s<d$, the Riesz $s$-energy of the sphere $\mathbb{S}^{d}$ has the explicit form

$$
W_{s}\left(\mathbb{S}^{d}\right)=\mathcal{I}_{s}\left[\sigma_{d}\right]=2^{d-1-s} \frac{\Gamma((d+1) / 2) \Gamma((d-s) / 2)}{\sqrt{\pi} \Gamma(d-s / 2)},
$$

expressed in terms of the gamma function $\Gamma$. By identifying $W_{s}\left(\mathbb{S}^{d}\right)$ with the analytic continuation of the right-hand side above to the complex $s$-plan 14 , we can define the Riesz $s$-energy of $\mathbb{S}^{d}$ for Riesz parameter $s$ for which the $s$-energy integral (3.4) is $+\infty$ for every Borel probability measure on $\mathbb{S}^{d}$. The combined effort of [43, 136, 167, 196, 197] resulted in the following bounds for the second term of the minimal energy asymptotics 15 there exist constants $c, C>0$ depending only on $d \geq 2$ and $0<s<d$ such that

$$
c N^{1+s / d} \leq \mathcal{E}_{s}\left(\mathbb{S}^{d} ; N\right)-W_{s}\left(\mathbb{S}^{d}\right) N^{2} \leq C N^{1+s / d}, \quad N \geq 2 .
$$

These estimates give the correct order of growth and sign for the second-order term. It is an open problem if the sequence $\left(\mathcal{E}_{s}\left(\mathbb{S}^{d} ; N\right)-W_{s}\left(\mathbb{S}^{d}\right) N^{2}\right) / N^{1+s / d}$ has a limit as $N \rightarrow \infty$. A. A. Berezin [24] used a semi-continuum approach (a classical method from solid state physics, cf. [101]) to derive the plausible asymptotics

$$
\begin{aligned}
\mathcal{E}_{s}\left(\mathbb{S}^{2} ; N\right) \approx N^{2} & \frac{2^{1-s}}{2-s}\left[1-(n / N)^{1-s / 2}\right]+N^{1+s / 2}\left(\frac{\sqrt{3}}{8 \pi}\right)^{s / 2} \\
& \times\left\{\frac{6}{1^{s}}+\frac{6}{(\sqrt{3})^{s}}+\frac{6}{2^{s}}+\frac{12}{(\sqrt{7})^{s}}+\frac{6}{3^{s}}+\frac{6}{(2 \sqrt{3})^{s}}+\frac{12}{(\sqrt{13})^{s}}+\cdots\right\}
\end{aligned}
$$

\footnotetext{
${ }^{14}$ The meromorphic function $W_{s}\left(\mathbb{S}^{d}\right)$, which appears in the conjecture for the asymptotics in the hypersingular case, has simple poles (finitely many if $d$ is even and infinitely many if $d$ is odd). The effect of this dichotomy on the asymptotic expansion of the minimal Riesz $s$-energy is completely open for $d \geq 2$ and leads to $\log N$ terms for the unit circle, cf. [57].

${ }^{15}$ Similar estimates but with negative constants $c, C$ hold for the sum of generalised distances (i.e., $-2<s<0$ ); see [2, 3, 4, 18, 125, 187, 188] and culminating in [196, 197].
} 
based on the assumptions that a typical point (and most of its immediate neighbours) in a minimal Riesz $s$-energy $N$-point configuration on $\mathbb{S}^{2}$ gives rise to a hexagonal Voronoi cell (sixfold symmetry) whereas the defects according to the curved surface of the sphere will have no significant influence on the second term in the asymptotics. Thus the contribution to the Riesz $s$-energy due to a typical point can be split into a local part which uses $n$ nearest neighbour points from a suitably adjusted flat hexagonal lattice and a distant part where the $N-n$ points are replaced by the continuous uniform distribution. The expression in curly braces gives the formal series expansion of the Epstein zeta function of the hexagonal lattices truncated to include only the $n-1$ shortest distances in the lattict 16 . (In order to get a non-trivial expansion, $n$ has to grow slowly in terms of $N$ to infinity. The paper 24] mentions some numerical experiments for slowly growing $n$ but a rigorous investigation has not been undertaken.) The discussion leading to Conjecture 1 below suggests that the semi-continuum approach would also work for $d=4$, 8, and 24 . In general, it is not clear which local approximation should be used.

For $\Omega$ in $\mathbb{R}^{p}$ with vanishing $s$-capacity, the leading term is rather well-understood. In the strictly hypersingular regime $s>d$, Hardin and Saff [116] (for rectifiable $d$-dimensional manifolds including the sphere $\mathbb{S}^{d}$ ) and Borodachov, Hardin, and Saff 34] (for infinite compact $d$-rectifiable set.17) established the existence of a constant $C_{s, d}$ such that for a large class of sets $\Omega 18$

$$
\lim _{N \rightarrow \infty} \frac{\mathcal{E}_{s}(\Omega ; N)}{N^{1+s / d}}=\frac{C_{s, d}}{\left[\mathcal{H}_{d}(\Omega)\right]^{s / d}},
$$

where $\mathcal{H}_{d}$ denotes the $d$-dimensional Hausdorff measure in $\mathbb{R}^{p}$ normalised such that the $d$ dimensional unit cube has $\mathcal{H}_{d}$-measure 1. This result is referred to as the Poppy-seed Bagel Theorem because of its interpretation for distributing points on a torus. Except for onedimensional sets (when $C_{s, 1}$ is twice the Riemann zeta function at $s$, see [150, Thm. 3.1]), the precise value of $C_{s, d}$ is not known. Its determination is a challenging open problem. The significance and difficulty of obtaining $C_{s, d}$ is due to the deep connection to densest packings. In [33] it is shown that $C_{s, d}$ is tied to the largest sphere packing density $\Delta_{d}$ in $\mathbb{R}^{d}$ and the best-packing distance $\delta_{N}^{*}$ of $N$-points on $\mathbb{S}^{d}$ by means of the limit relation 19

$$
\lim _{s \rightarrow \infty}\left[C_{s, d}\right]^{-1 / s}=2\left[\frac{\Delta_{d}}{\mathcal{H}_{d}\left(\mathbb{B}^{d}\right)}\right]^{1 / d}=\lim _{N \rightarrow \infty} N^{1 / d} \delta_{N}^{*} .
$$

Here, $\mathcal{H}_{d}\left(\mathbb{B}^{d}\right)$ is the volume of the unit ball in $\mathbb{R}^{d}$. We recall that $\Delta_{d}$ is only known for three cases: $\Delta_{1}=1, \Delta_{2}=\pi / \sqrt{12}$ (Thue [192] and L. Fejes Tóth [92]) and $\Delta_{3}=\pi / \sqrt{18}$ (Kepler conjecture proved by Hales [110]). The connection to (regular) lattices is evident in the upper estimate of the constant $C_{s, d}$ in terms of the Epstein zeta function $\zeta_{\Lambda}(s)$ of

\footnotetext{
${ }^{16}$ Indeed, the first few most frequent distances in a putative minimal energy configuration emulate remarkably well the first few distances in a hexagonal lattice (see, in particular, 59, Figure 1]).

${ }^{17} \mathrm{~A} d$-rectifiable set is the Lipschitz image of a bounded set in $\mathbb{R}^{d}$.

${ }^{18}$ The boundedness of $\mathcal{E}_{s}\left(\mathbb{S}^{d} ; N\right) / N^{1+s / d}$ has already been shown in [136].

${ }^{19}$ Indeed, one can recast this relation as $\Delta_{d}=\lim _{s \rightarrow \infty} \lim _{N \rightarrow \infty}\left[\mathcal{E}_{s}\left(\frac{1}{2} \mathbb{B}^{d} ; N\right) / N^{1+s / d}\right]^{-d / s}$.
} 
a lattice $\Lambda$ in $\mathbb{R}^{d}$ which can be obtained by considering the Riesz s-energy of the $N=n^{d}$ points of the rescaled lattice $\frac{1}{n} \Lambda$ lying in the fundamental parallelotope $\Omega$ of $\Lambda$ and which implies that $\mathcal{E}_{s}(\Omega ; N) \leq n^{d+s} \zeta_{\Lambda}(s)=N^{1+s / d} \zeta_{\Lambda}(s)$ and thus for $s>d$ (cf. [57, Prop. 1]),

$$
C_{s, d} \leq \min _{\Lambda}|\Lambda|^{s / d} \zeta_{\Lambda}(s)
$$

where the minimum is extended over all lattices $\Lambda$ in $\mathbb{R}^{d}$ with positive co-volume $|\Lambda|$. Because of (3.14), the sharpness of this inequality touches on questions regarding densest lattice sphere packings. For $1 \leq d \leq 8$ and $d=24$, the unique densest lattice in $\mathbb{R}^{d}$ up to scaling and isometries is the root lattice $A_{1}, A_{2}, A_{3}, D_{4}, D_{5}, E_{6}, E_{7}, E_{8}$, and the Leech lattice $\Lambda_{24}$, respectively (cf. [75]). Among those the hexagonal lattice $A_{2}$ in $\mathbb{R}^{2}$, the $E_{8}$ root lattice in $\mathbb{R}^{8}$ and the Leech lattice $\Lambda_{24}$ in $\mathbb{R}^{24}$ are conjectured to be universally optimal, whereas the remaining lattices are provably not universally optimal (cf. [74, 76]). See [176] for local optimality results and [81] for improvements. Montgomery [155] proved that the hexagonal lattice is universally optimal amongst all lattices in $\mathbb{R}^{2}$ (which is weaker than universal optimality amongst all periodic point configurations). H. Cohn and N. Elkies [73] conjectured that $\mathrm{E}_{8}$ and the Leech lattice $\Lambda_{24}$ solve the sphere packing problem in their dimension. It is generally expected that for sufficiently large $d$, lattice packings are not densest packings and 193] suggests that best-packings are highly "disordered" as $d \rightarrow \infty$. This motivates the following conjecture20.

Conjecture 1 ([57]). For $d=2,4,8$, and 24 , one has $C_{s, d}=\left|\Lambda_{d}\right|^{s / d} \zeta_{\Lambda_{d}}(s)$, where $\Lambda_{d}$ denotes, respectively, the hexagonal lattice $\mathrm{A}_{2}$, the root lattices $\mathrm{D}_{4}$ and $\mathrm{E}_{8}$, and the Leech lattice $\Lambda_{24}$.

We remark that in [59] very coarse lower and upper bounds are obtained for $\mathcal{E}_{s}\left(\mathbb{S}^{d} ; N\right)$ which imply for $s>d \geq 2$ and $(s-d) / 2$ not an integer, the estimates

$$
\frac{d}{s-d}\left[\frac{1}{2} \frac{\Gamma((d+1) / 2) \Gamma(1+(s-d) / 2)}{\sqrt{\pi} \Gamma(1+s / 2)}\right]^{s / d} \leq \frac{C_{s, d}}{\left[\mathcal{H}_{d}\left(\mathbb{S}^{d}\right)\right]^{s / d}} \leq\left[\frac{\mathcal{H}_{d}\left(\mathbb{B}^{d}\right)}{\mathcal{H}_{d}\left(\mathbb{S}^{d}\right)(1-d / s)}\right]^{s / d} .
$$

In the hypersingular case $s=d$, more can be said. It has been known from [136] that the leading term of $\mathcal{E}_{d}\left(\mathbb{S}^{d} ; N\right)$ grows like $N^{2} \log N$ and

$$
\lim _{N \rightarrow \infty} \frac{\mathcal{E}_{d}\left(\mathbb{S}^{d} ; N\right)}{N^{2} \log N}=\frac{\mathcal{H}_{d}\left(\mathbb{B}^{d}\right)}{\mathcal{H}_{d}\left(\mathbb{S}^{d}\right)}=\frac{1}{d} \frac{\omega_{d-1}}{\omega_{d}}=\frac{1}{d} \frac{\Gamma((d+1) / 2)}{\sqrt{\pi} \Gamma(d / 2)} .
$$

The best estimates so far for the second-order term has been obtained recently in [59],

$$
-c(d) N^{2}+\mathcal{O}\left(N^{2-2 / d} \log N\right) \leq \mathcal{E}_{d}\left(\mathbb{S}^{d} ; N\right)-\frac{\mathcal{H}_{d}\left(\mathbb{B}^{d}\right)}{\mathcal{H}_{d}\left(\mathbb{S}^{d}\right)} N^{2} \log N \leq \frac{\mathcal{H}_{d}\left(\mathbb{B}^{d}\right)}{\mathcal{H}_{d}\left(\mathbb{S}^{d}\right)} N^{2} \log \log N+\mathcal{O}\left(N^{2}\right)
$$

\footnotetext{
${ }^{20}$ The conjecture for $d=2$ appeared in 136 .
} 
as $N \rightarrow \infty$, where the constant $c(d)$ is given by

$$
c(2)=1 / 2, \quad c(d):=\frac{\mathcal{H}_{d}\left(\mathbb{B}^{d}\right)}{\mathcal{H}_{d}\left(\mathbb{S}^{d}\right)}\left\{1-\log \frac{\mathcal{H}_{d}\left(\mathbb{B}^{d}\right)}{\mathcal{H}_{d}\left(\mathbb{S}^{d}\right)}+d[\psi(d / 2)-\psi(1)-\log 2]\right\}>0 .
$$

(Recall, that $\psi=\psi^{\prime} / \psi$ denotes the digamma function.) Based on a limiting process $s \rightarrow d$ in Conjecture 2 given below, it is conjectured in [59] that the correct order of the second term is $N^{2}$. Furthermore, in the case $d=2$ a conjecture is posed for the constant of the $N^{2}$-term.

\subsection{Higher Order Terms - Complete Asymptotic Expansions - Fundamental Conjecture}

Very little is known about higher-order terms of the asymptotics of the minimal Riesz $s$-energy except for the unit circle. As the $N$ th roots of unity are universally optimal 21 the complete asymptotic expansion can be obtained by direct computation of the Riesz $s$ energy $\mathcal{L}_{s}(N)$ of the $N$ th roots of unity, see [57] for Euclidean and [58] for geodesic metric. Indeed, for $s \in \mathbb{C}$ with $s \neq 0,1,3,5, \ldots$ and fixed $p=1,2,3, \ldots$, one hat 22

$$
\begin{aligned}
\mathcal{L}_{s}(N)= & W_{s}\left(\mathbb{S}^{1}\right) N^{2}+\frac{2 \zeta(s)}{(2 \pi)^{s}} N^{1+s}+\sum_{n=1}^{p} \alpha_{n}(s) \frac{2 \zeta(s-2 n)}{(2 \pi)^{s}} N^{1+s-2 n} \\
& +\mathcal{O}_{s, p}\left(N^{-1+\operatorname{Re}(s)-2 p}\right) \quad \text { as } N \rightarrow \infty
\end{aligned}
$$

where the coefficients $\alpha_{n}(s), n \geq 0$, are defined by the generating function relation

$$
\left(\frac{\sin \pi z}{\pi z}\right)^{-s}=\sum_{n=0}^{\infty} \alpha_{n}(s) z^{2 n}, \quad|z|<1, s \in \mathbb{C}
$$

Explicit formulas for $\alpha_{n}(s)$ in terms of generalised Bernoulli polynomials $B_{n}^{(\alpha)}(x)$ are given in [58]. The asymptotics (3.17) has two noteworthy features: this expansion is valid for complex $s$ and the Riemann zeta function plays an essential role. The coefficients of the terms in the asymptotics are best understood as functions in the complex $s$-plane. This is called the principle of analytic continuation 23 The interplay between the simple poles of the coefficient $W_{s}\left(\mathbb{S}^{1}\right)$ of the $N^{2}$-term in (3.12) and the simple poles of the shifted Riemann zeta functions then gives rise to a logarithmic term whenever $s$ tends to one of the exceptional cases $s=0,1,3,5, \ldots$.

By combining the results for the potential theoretic and the hypersingular regime, the principle of analytic continuation motivates the following fundamental conjecture.

\footnotetext{
${ }^{21}$ For $s \geq-1$ (and $s \neq 0$ ) a convexity argument can be applied to get optimality for Riesz $s$-energy (see [4, 91, 99]). The much more general result [74, Theorem 1.2] provides optimality for $s>-2$.

${ }^{22}$ The precise formulas for finite $N \geq 2$ are obtained in [46].

${ }^{23}$ This principle breaks down when the perfectly symmetric unit circle is replaced by some other smooth closed curve $\Gamma$. Then the $s$-equilibrium measure on $\Gamma$ is not the normalised arc-length measure for each $0<s<1$ which plays a role in the characterization of the coefficient of $N^{2}$ in the hypersingular regime $1<s<3$; see 31 .
} 
Conjecture 2 (see [59]). Let $d \geq 2$. Then for $0<s<d+2$ with $s \neq d$,

$$
\mathcal{E}_{s}\left(\mathbb{S}^{d} ; N\right)=W_{s}\left(\mathbb{S}^{d}\right) N^{2}+\frac{C_{d}(s)}{\left[\mathcal{H}_{d}\left(\mathbb{S}^{d}\right)\right]^{s / d}} N^{1+s / d}+o\left(N^{1+s / d}\right) \quad \text { as } N \rightarrow \infty,
$$

where $W_{s}\left(\mathbb{S}^{d}\right)$ is the analytic continuation of the right-hand side of (3.12) and $C_{d}(s)$ is the analytic continuation of $C_{s, d}$ in (3.13). Furthermore, for $d=2,4,8$, and 24 , the constant $C_{d}(s)$ is the analytic continuation of $\left|\Lambda_{d}\right|^{s / d} \zeta_{\Lambda_{d}}(s)$, where $\Lambda_{d}$ is given in Conjecture 1 .

It should be discussed briefly that the asymptotic expansion of the minimal s-energy can also be studied from a geometrical point of view by identifying which features of the Voronoi cell decomposition (or its dual, the Delaunay triangulation) induced by minimal $s$-energy configurations contribute in which way to the asymptotics. For the 2 -sphere and large $N$, the typical picture is a vast sea of hexagonal Voronoi cells - thus the local approximation of the neighbourhood of a typical point is done by a suitably scaled hexagonal lattice to get the second term of the asymptotics. The topology of the sphere gives rise to geometric frustration (cf. [172]) where certain points pick up a topological charge that measures the discrepancy from the ideal coordination number (six) of the planar triangular lattice. Euler's celebrated Polyhedral formula yields that the total topological charge on $\mathbb{S}^{2}$ is always 12 . Numerically, one observes "scars" for large $N$ emerging from 12 pentagonal centres. These scars attract pentagon-heptagon pairs which have total topological charge zero. It is an unresolved question if there are Voronoi cells with more than 7 sides in minimising configurations. It is not well understood how (i.e., on which level of the asymptotic scale) scars, the type of Voronoi cells, and the variation in their sizes affect higher-order terms of the asymptotics; see [65] for numerical background and [41] for an approach using elastic continuum formalism.

\subsection{Asymptotic expansion of logarithmic energy}

The leading term of the asymptotic expansion for a compact set $\Omega$ in $\mathbb{R}^{p}$ with positive logarithmic capacity (i.e., finite logarithmic energy) follows from classical potential theory. It should be noted that (see [36])

$$
\left.\frac{\mathrm{d}}{\mathrm{d} s} \mathcal{E}_{s}(\Omega ; N)\right|_{s=0^{+}}=\mathcal{E}_{\log }(\Omega ; N), \quad N \geq 2 .
$$

For the unit sphere $\mathbb{S}^{d}$, one has

$$
\lim _{N \rightarrow \infty} \frac{\mathcal{E}_{\log }\left(\mathbb{S}^{d} ; N\right)}{N^{2}}=W_{\log }\left(\mathbb{S}^{d}\right)=\log \frac{1}{\operatorname{cap}_{\log }\left(\mathbb{S}^{d}\right)},
$$

where the logarithmic energy of $\mathbb{S}^{d}$ is given by

$$
W_{\log }\left(\mathbb{S}^{d}\right)=\left.\frac{\mathrm{d} W_{s}\left(\mathbb{S}^{d}\right)}{\mathrm{d} s}\right|_{s=0^{+}}=\log \frac{1}{2}+\frac{1}{2}[\psi(d)-\psi(d / 2)] .
$$


Here, $\psi(z)$ is the digamma function. An averaging argument that uses an equal-area partition of $\mathbb{S}^{d}$ and bounds of G. Wagner [197] and [44] yields

$$
\mathcal{E}_{\log }\left(\mathbb{S}^{d} ; N\right)=W_{\log }\left(\mathbb{S}^{d}\right) N^{2}-\frac{1}{d} N \log N+\mathcal{O}(N), \quad N \rightarrow \infty .
$$

Relation (3.18) and Conjecture2 provide the basis for the following conjecture posed in [59].

Conjecture 3. For $d=2,4,8$, and 24,

$$
\mathcal{E}_{\log }\left(\mathbb{S}^{d} ; N\right)=W_{\log }\left(\mathbb{S}^{d}\right) N^{2}-\frac{1}{d} N \log N+C_{\log , d} N+o(N) \quad \text { as } N \rightarrow \infty,
$$

where

$$
C_{\log , d}=\frac{1}{d} \log \frac{\mathcal{H}_{d}\left(\mathbb{S}^{d}\right)}{\left|\Lambda_{d}\right|}+\zeta_{\Lambda_{d}}^{\prime}(0)
$$

For $d=2$ one has

$$
C_{\log , 2}=2 \log 2+\frac{1}{2} \log \frac{2}{3}+3 \log \frac{\sqrt{\pi}}{\Gamma(1 / 3)}=-0.05560530494339251850 \ldots
$$

For more details see [59]. Very recently, L. Bétermin [25] found a surprising connection between the problem of minimising a planar "Coulombian renormalised energy" derived from the Ginzburg-Landau model of superconductivity introduced by E. Sandier and S. Serfaty in [175] (also see the survey [180]) and the discrete logarithmic energy problem on $\mathbb{S}^{2}$. The preliminary results are: (i) the asymptotics of the minimal logarithmic energy on $\mathbb{S}^{2}$ has a term of order $N$, (ii) whose constant is bounded from above by $C_{\log , 2}$ given above, and (iii) this constant equals $C_{\log , 2}$ if and only if a certain triangular lattice of density 1 (called "Abrikosov" triangular lattice $\mathbb{Z}+e^{i \pi / 3} \mathbb{Z}$, properly scaled, see [180]) is the minimiser of the Coulombian renormalised energy.

\subsection{Numerical integration and discrepancy from the energy point of view}

The reproducing kernel Hilbert space approach (see Section 2) enables us to write the squared worst-case error as

$$
\frac{1}{N^{2}} \sum_{i=1}^{N} \sum_{j=1}^{N} \mathrm{~K}\left(\mathbf{x}_{i}, \mathbf{x}_{j}\right)-\int_{\mathbb{S}^{d}} \int_{\mathbb{S}^{d}} \mathrm{~K}(\mathbf{x}, \mathbf{y}) \mathrm{d} \sigma_{d}(\mathbf{x}) \mathrm{d} \sigma_{d}(\mathbf{y})
$$

which can be interpreted as K-energy of the node set $\left\{\mathbf{x}_{1}, \ldots, \mathbf{x}_{N}\right\}$ of the QMC method. Here, the energy kernel is a reproducing kernel for $H^{s}\left(\mathbb{S}^{d}\right)$. Optimal node sets are solutions of the minimal energy problem for this kernel. In [48] and [49] it is shown how the distance kernel

$$
\mathrm{K}_{\mathrm{gd}}^{(s)}(\mathbf{x}, \mathbf{y}):=2 W_{d-2 s}\left(\mathbb{S}^{d}\right)-\|\mathbf{x}-\mathbf{y}\|^{2 s-d}, \quad \mathbf{x}, \mathbf{y} \in \mathbb{S}^{d},
$$

arises in a natural way as a reproducing kernel for $H^{s}\left(\mathbb{S}^{d}\right)$ for $s$ in $(d / 2, d / 2+1)$. Evidently, there is a close connection between finding optimal QMC nodes and the problem of 
maximizing the sum of all pairwise distances taken to the power $2 s-d$. Wagner's bounds (see [196, 197]) yield that a sequence of $N$-point maximisers of such a generalised sum of distances is a QMC design sequence for $H^{s}\left(\mathbb{S}^{d}\right), d / 2<s<d / 2+1$; see [61]. Explicit constructions for QMC design sequences are not known. Based on numerical evidence it is conjectured in [1, 47] that a $(0,2)$-sequence (a special digital net sequence in the sense of [85]) or a Fibonacci lattice (also see [85]) in the square $[0,1)^{2}$ mapped to the 2-sphere via an area-preserving map (Lambert azimuthal equal-area projection) will be a QMC design sequence for $H^{3 / 2}\left(\mathbb{S}^{2}\right)$. For $s \geq d / 2+1$, the space $H^{s}\left(\mathbb{S}^{d}\right)$ can also be provided with a reproducing kernel which is essentially a distance kernel with power $2 s-d$. In order to ensure that the reproducing kernel is positive definite (in the sense of Schoenberg [178]) a polynomial correction term is needed when $s-d / 2$ is not a positive integer 2425 Such a correction term is annihilated when the search for optimal QMC designs for $H^{s}\left(\mathbb{S}^{d}\right)$, $s \in(d / 2+L, d / 2+1+L)$, is restricted to spherical $L$-designs. In that case it suffices to minimise the energy functional (see [61])

$$
\left[\operatorname{wce}_{H^{s}}\left(X_{N, L}\right)\right]^{2}=\frac{1}{N^{2}} \sum_{i=1}^{N} \sum_{j=1}^{N}(-1)^{L+1}\left\|\mathbf{x}_{i, L}-\mathbf{x}_{j, L}\right\|^{2 s-d}-(-1)^{L+1} W_{d-2 s}\left(\mathbb{S}^{d}\right)
$$

subject to the condition that the node set $X_{N, L}=\left\{\mathbf{x}_{1, L}, \ldots, \mathbf{x}_{N, L}\right\} \subset \mathbb{S}^{d}$ is a spherical $L$-design. Note that the fixed $L$ (once $s$ is fixed) is "small" in the following sense: a QMC method with a more regular (in the sense of spherical designs) node set is more suitable for integrating functions from a smoother function space $H^{s}\left(\mathbb{S}^{d}\right)$. But regularity beyond a critical order will not improve the worst-case error bound; this should be compared with the optimal order (2.37). We remark that the Cui and Freeden kernel [82]

$$
\mathrm{K}_{\mathrm{CF}}(\mathbf{x}, \mathbf{y}):=2-2 \log \left(1+\frac{1}{2}\|\mathbf{x}-\mathbf{y}\|\right), \quad \mathbf{x}, \mathbf{y} \in \mathbb{S}^{2},
$$

which was used to define a "generalised discrepancy" to measure uniform distribution of point set sequences, can be interpreted as reproducing kernel for $H^{3 / 2}\left(\mathbb{S}^{2}\right)$ as observed in [184] and the minimising $\mathrm{K}_{\mathrm{CF}^{-}}$-energy point configurations give rise to a $\mathrm{QMC}$ design sequence for $H^{3 / 2}\left(\mathbb{S}^{2}\right)$. Recently, C. Choirat and R. Seri [69, 70] derived the analogous kernel for $d$-spheres. The corresponding minimising configurations then form QMC design sequences for $H^{(d+1) / 2}\left(\mathbb{S}^{d}\right)$. A curious observation is that when $H^{s}\left(\mathbb{S}^{d}\right), d / 2<s<d / 2+1$, is provided with the reproducing kernel $\mathrm{K}_{\mathrm{gd}}^{(s)}$ from (3.19), a limit process yields that (see [62])

$$
\lim _{s \rightarrow(d / 2)^{+}} \frac{\left[\operatorname{wce}_{H^{s}}\left(X_{N}\right)\right]^{2}-\frac{1}{N}}{2 s-d}=\frac{1}{N^{2}} \sum_{i=1}^{N} \sum_{\substack{j=1 \\ i \neq j}}^{N} \log \frac{1}{\left\|\mathbf{x}_{i}-\mathbf{x}_{j}\right\|}-V_{\log }\left(\mathbb{S}^{d}\right)
$$

\footnotetext{
${ }^{24} \mathrm{~A}$ logarithm of the distance appears in the reproducing kernel when $s-d / 2$ is a positive integer.

${ }^{25}$ When defining the function space as Bessel potential space, then no correction terms are needed. In the Hilbert space setting $(p=2)$, the worst-case is given as Bessel-energy. The Bessel kernel on the sphere, however, has a series expansion in spherical harmonics without convenient closed form representation. For general $p>1$ the worst-case error has an integral representation; see [51] and also [42].
} 
for any $N$-point set $X_{N}=\left\{\mathbf{x}_{1}, \ldots, \mathbf{x}_{N}\right\} \subset \mathbb{S}^{d}$. This suggests that the function space $H^{d / 2}\left(\mathbb{S}^{d}\right)$ (which is not a reproducing kernel Hilbert space) is paired with the logarithmic kernel and the logarithmic energy of an $N$-point set can be understood as a limit of worstcase errors in the above sense. This paring can be extended to $s<d / 2$ and the open question is how to define integration and error of integration so that the corresponding Riesz $(2 s-d)$-energy is a meaningful measure for this error.

Due to J. Beck [18], the spherical cap discrepancy $D\left(X_{N}\right)$ given in (2.16) of any $X_{N} \subset \mathbb{S}^{d}$ is bounded like $\gg N^{-1 / 2-1 /(2 d)}$ and, by appealing to a probabilistic argument, there are $X_{N} \subset \mathbb{S}^{d}$ with

$$
D\left(X_{N}\right) \ll N^{-1 / 2-1 /(2 d)} \sqrt{\log N} .
$$

A sequence of point sets with this property is called a low-discrepancy sequence on $\mathbb{S}^{d}$. It is shown in [61] that a low-discrepancy sequence $\left(X_{N}^{\mathrm{LD}}\right)$ on $\mathbb{S}^{d}$ is almost a QMC design sequence for $H^{(d+1) / 2}\left(\mathbb{S}^{d}\right)$ in the sense that for sufficiently large $N$,

$$
c N^{-(d+1) /(2 d)} \leq \operatorname{wce}_{H^{(d+1) / 2}}\left(X_{N}^{\mathrm{LD}}\right) \leq C N^{-(d+1) /(2 d)} \sqrt{\log N} .
$$

One of the deep unresolved questions is if the logarithmic term in (3.20) arising from a probabilistic argument can be removed. It is also unknown how to construct a sequence of $N$-point sets explicitly with spherical cap discrepancy decaying like $N^{-1 / 2-1 /(2 d)} \sqrt{\log N}$. For the 2-sphere, the construction of A. Lubotzky, R. Phillips, and P. Sarnak 146, 147] satisfies the estimate $D\left(X_{N}^{\mathrm{LPS}}\right) \ll(\log N)^{2 / 3} N^{-1 / 3}$ with numerical evidence indicating a convergence rate of $\mathcal{O}\left(N^{-1 / 2}\right)$, whereas the spherical Fibonacci lattice point sets of [1] obey the estimate $D\left(X_{N}^{A B D}\right) \leq 44 \sqrt{8} N^{-1 / 2}$ with numerical results showing a convergence rate of $\mathcal{O}\left((\log N)^{c} N^{-3 / 4}\right)$ for some $1 / 2 \leq c \leq 1$. The typical spherical cap discrepancy of $N$ i.i.d. uniformly distributed random points on $\mathbb{S}^{2}$ is of exact order $N^{-1 / 2}$, see [1]. Surprisingly, minimal Coulomb energy points on $\mathbb{S}^{2}$ do not have low spherical cap discrepancy. J. Korevaar [132] conjectured that minimal $(d-1)$-energy configurations on $\mathbb{S}^{d}$ have spherical cap discrepancy of order $N^{-1 / d}$. This conjecture was proven by M. Götz [98] up to a logarithmic factor. He also gave a lower bound of order $N^{-1 / 2}$ for $d=2$. It is open if Korevaar's conjecture extends to the full potential-theoretic regime. On the basis of the Poppy-seed Bagel theorem, one could conjecture that minimal s-energy points for hypersingular $s$ should have small spherical cap discrepancy. The only result obtained so far is the very weak order $\sqrt{\log \log N} / \log N$ result in [83] for the boundary case $s=d$. The proof employs a smoothened Riesz energy functional.

Stolarsky's invariance principle (see K. B. Stolarsky [188]) states that the sum of all mutual distances (a Riesz energy with Riesz parameter -1) and the spherical cap $L^{2}$ discrepancy

$$
D_{L^{2}}\left(X_{N}\right):=\left(\int_{0}^{\pi} \int_{\mathbb{S}^{d}}\left|\frac{1}{N} \sum_{\mathbf{y} \in X_{N}} \mathbb{1}_{\mathbf{y} \in C(\mathbf{z}, \theta)}-\sigma_{d}(C(\mathbf{z}, \theta))\right|^{2} \mathrm{~d} \sigma_{d}(\mathbf{z}) \sin \theta \mathrm{d} \theta\right)^{1 / 2}
$$


is constant regardless of the choice of the node set $X_{N}$ on $\mathbb{S}^{d}$; i.e.,

$$
\frac{1}{N^{2}} \sum_{i=1}^{N} \sum_{j=1}^{N}\left\|\mathbf{x}_{i}-\mathbf{x}_{j}\right\|+\frac{1}{C_{d}}\left[D_{L^{2}}\left(X_{N}\right)\right]^{2}=\int_{\mathbb{S}^{d}} \int_{\mathbb{S}^{d}}\|\mathbf{x}-\mathbf{y}\| \mathrm{d} \sigma_{d}(\mathbf{x}) \mathrm{d} \sigma_{d}(\mathbf{y}) .
$$

This principle connects in a very direct way the three areas optimal energy (maximizing the sum of distances on $\mathbb{S}^{d}$ ), uniform distribution (spherical cap $L^{2}$-discrepancy), and numerical integration with QMC methods for functions on $\mathbb{S}^{d}$. Rearranging terms,

$$
\frac{1}{\sqrt{C_{d}}} D_{L^{2}}\left(X_{N}\right)=\sqrt{\int_{\mathbb{S}^{d}} \int_{\mathbb{S}^{d}}\|\mathbf{x}-\mathbf{y}\| \mathrm{d} \sigma_{d}(\mathbf{x}) \mathrm{d} \sigma_{d}(\mathbf{y})-\frac{1}{N^{2}} \sum_{i=1}^{N} \sum_{j=1}^{N}\left\|\mathbf{x}_{i}-\mathbf{x}_{j}\right\|}
$$

one obtains a convenient way of computing the discrepancy $D_{L^{2}}\left(X_{N}\right)$. It is shown in [49] that the right-hand side represents the worst-case error of a QMC method with node set $\left\{\mathbf{x}_{1}, \ldots, \mathbf{x}_{N}\right\} \subset \mathbb{S}^{d}$ for functions in $H^{s}\left(\mathbb{S}^{d}\right)$ provided with the distance kernel $\mathrm{K}_{\mathrm{gd}}^{(s)}$ given in (3.19) with $s=(d+1) / 2$. Moreover, in this setting, the spherical cap $L^{2}$-discrepancy can be interpreted as worst-case error and vice versa. A conjecture for the asymptotic expansion (as $N \rightarrow \infty$ ) of the minimal spherical cap $L^{2}$-discrepancy that is based on the fundamental conjecture for the minimal Riesz $s$-energy and the principle of analytic continuation is proposed in [45]. The paper [48] gives an extension of Stolarsky's invariance principle to general powers of the distance (raised to the power $2 s-d$ ) involving the generalised spherical $L_{2}$-discrepancy

$$
\int_{0}^{\pi} \int_{\mathbb{S}^{d}}\left|\mathcal{D}_{X_{N}, \beta}(\mathbf{z} ; \cos \theta)\right|^{2} \mathrm{~d} \sigma_{d}(\mathbf{z}) \sin \theta \mathrm{d} \theta
$$

for the local discrepancy function (with smoothness index $s=\beta+(d-1) / 2$ )

$$
\mathcal{D}_{X_{N}, \beta}(\mathbf{z} ; t):=\frac{1}{N} \sum_{\mathbf{x} \in X_{N}}(\mathbf{x} \cdot \mathbf{z}-t)_{+}^{\beta-1}-\int_{\mathbb{S}^{d}}(\mathbf{y} \cdot \mathbf{z}-t)_{+}^{\beta-1} \mathrm{~d} \sigma_{d}(\mathbf{y}) .
$$

The paper [50] considers the discrepancy with respect to truncated spherical cones that are anchored at infinity and extends Stolarsky's invariance principle to this setting. In 103. further connections between energy and discrepancy are discussed. We also mention [143] which considers asymptotically uniformly distributed points with an upper bound on the spherical cap discrepancy and a lower bound on the separation.

Acknowledgement. The authors are indebted to three anonymous referees for their valuable remarks and suggestions which greatly increased the quality of presentation of this paper. They are grateful to Wöden Kusner for his careful proofreading of the manuscript and his many invaluable comments. 


\section{References}

[1] Aistleitner, C., Brauchart, J. S., Dick, J., 2012. Point Sets on the Sphere $\mathbb{S}^{2}$ with Small Spherical Cap Discrepancy. Discrete Comput. Geom. 48 (4), 990-1024.

[2] Alexander, R., 1972. On the sum of distances between $n$ points on a sphere. Acta Math. Acad. Sci. Hungar. 23, 443-448.

[3] Alexander, R., 1977. On the sum of distances between $n$ points on a sphere. II. Acta Math. Acad. Sci. Hungar. 29 (3-4), 317-320.

[4] Alexander, R., Stolarsky, K. B., 1974. Extremal problems of distance geometry related to energy integrals. Trans. Amer. Math. Soc. 193, 1-31.

[5] Ambrus, G., Ball, K. M., Erdélyi, T., 2013. Chebyshev constants for the unit circle. Bull. Lond. Math. Soc. 45 (2), 236-248.

[6] An, C., Chen, X., Sloan, I. H., Womersley, R. S., 2010. Well conditioned spherical designs for integration and interpolation on the two-sphere. SIAM J. Numer. Anal. 48 (6), 2135-2157.

[7] An, C., Chen, X., Sloan, I. H., Womersley, R. S., 2012. Regularized least squares approximations on the sphere using spherical designs. SIAM J. Numer. Anal. 50 (3), $1513-1534$.

[8] Apostol, T. M., 1990. Modular functions and Dirichlet series in number theory, 2nd Edition. Vol. 41 of Graduate Texts in Mathematics. Springer-Verlag, New York.

[9] Armentano, D., Beltrán, C., Shub, M., 2011. Minimizing the discrete logarithmic energy on the sphere: the role of random polynomials. Trans. Amer. Math. Soc. 363 (6), 2955-2965.

[10] Bachoc, C., 2005. Designs, groups and lattices. J. Théor. Nombres Bordeaux 17 (1), 25-44.

[11] Bachoc, C., Vallentin, F., 2008. New upper bounds for kissing numbers from semidefinite programming. J. Amer. Math. Soc. 21 (3), 909-924.

[12] Bachoc, C., Venkov, B., 2001. Modular forms, lattices and spherical designs. In: Réseaux euclidiens, designs sphériques et formes modulaires. Vol. 37 of Monogr. Enseign. Math. Enseignement Math., Geneva, pp. 87-111.

[13] Ballinger, B., Blekherman, G., Cohn, H., Giansiracusa, N., Kelly, E., Schürmann, A., 2009. Experimental study of energy-minimizing point configurations on spheres. Experiment. Math. 18 (3), 257-283.

[14] Bannai, E., 1987. Rigid spherical $t$-designs and a theorem of Y. Hong. J. Fac. Sci. Univ. Tokyo Sect. IA Math. 34, 485-489. 
[15] Bannai, E., Bannai, E., 2009. A survey on spherical designs and algebraic combinatorics on spheres. European J. Combin. 30 (6), 1392-1425.

[16] Bannai, E., Damerell, R. M., 1979. Tight spherical designs. I. J. Math. Soc. Japan 31 (1), 199-207.

[17] Bannai, E., Damerell, R. M., 1980. Tight spherical designs. II. J. London Math. Soc. (2) 21 (1), 13-30.

[18] Beck, J., 1984. Sums of distances between points on a sphere -an application of the theory of irregularities of distribution to discrete geometry. Mathematika 31 (1), $33-41$.

[19] Beltrán, C., 2013. Harmonic properties of the logarithmic potential and the computability of elliptic Fekete points. Constr. Approx. 37 (1), 135-165.

[20] Beltrán, C., 2013. The state of the art in Smale's 7th problem. In: Foundations of computational mathematics, Budapest 2011. Vol. 403 of London Math. Soc. Lecture Note Ser. Cambridge Univ. Press, Cambridge, pp. 1-15.

[21] Beltrán, C., 2015. A facility location formulation for stable polynomials and elliptic Fekete points. Found. Comput. Math.To appear.

[22] Bendito, E., Carmona, A., Encinas, A. M., Gesto, J. M., Gómez, A., Mouriño, C., Sánchez, M. T., 2009. Computational cost of the Fekete problem. I. The forces method on the 2-sphere. J. Comput. Phys. 228 (9), 3288-3306.

[23] Berens, H., Butzer, P. L., Pawelke, S., 1968/1969. Limitierungsverfahren von Reihen mehrdimensionaler Kugelfunktionen und deren Saturationsverhalten. Publ. Res. Inst. Math. Sci. Ser. A 4, 201-268.

[24] Berezin, A. A., 1986. Asymptotics of the maximum number of repulsive particles on a spherical surface. J. Math. Phys. 27 (6), 1533-1536.

[25] Bétermin, L., 2014. Renormalized energy and asymptotic expansion of optimal logarithmic energy on the sphere, manuscript, arXiv:1404.4485v2 [math.AP].

[26] Björck, G., 1956. Distributions of positive mass, which maximize a certain generalized energy integral. Ark. Mat. 3, 255-269.

[27] Blum, L., Cucker, F., Shub, M., Smale, S., 1997. Complexity and real computation. Foreword by Richard M. Karp. New York, NY: Springer.

[28] Bondarenko, A. V., Hardin, D. P., Saff, E. B., 2014. Mesh ratios for best-packing and limits of minimal energy configurations. Acta Math. Hung. 142 (1), 118-131.

[29] Bondarenko, A. V., Radchenko, D., Viazovska, M. S., 2013. Optimal asymptotic bounds for spherical designs. Ann. of Math. (2) 178 (2), 443-452. 
[30] Bondarenko, A. V., Radchenko, D., Viazovska, M. S., 2015. Well separated spherical designs. Constr. Approx.To appear.

[31] Borodachov, S. V., 2012. Lower order terms of the discrete minimal Riesz energy on smooth closed curves. Canad. J. Math. 64 (1), 24-43.

[32] Borodachov, S. V., Bosuwan, N., 2014. Asymptotics of Discrete Riesz d-Polarization on Subsets of d-Dimensional Manifolds. Potential Anal. 41 (1), 35-49.

[33] Borodachov, S. V., Hardin, D. P., Saff, E. B., 2007. Asymptotics of best-packing on rectifiable sets. Proc. Amer. Math. Soc. 135 (8), 2369-2380 (electronic).

[34] Borodachov, S. V., Hardin, D. P., Saff, E. B., 2008. Asymptotics for discrete weighted minimal Riesz energy problems on rectifiable sets. Trans. Amer. Math. Soc. 360 (3), 1559-1580 (electronic).

[35] Borodachov, S. V., Hardin, D. P., Saff, E. B., 2014. Low complexity methods for discretizing manifolds via riesz energy minimization. Found. Comput. Math., 1-36.

[36] Borodachov, S. V., Hardin, D. P., Saff, E. B., 2015. Minimal Discrete Energy on the Sphere and Other Manifolds, Springer Verlag, to appear.

[37] Borodin, A., Serfaty, S., 2013. Renormalized energy concentration in random matrices. Comm. Math. Phys. 320 (1), 199-244.

[38] Bowick, M., Cacciuto, A., Nelson, D. R., Travesset, A., 2002. Crystalline order on a sphere and the generalized Thomson problem. Phys. Rev. Lett. 89, 185502.

[39] Bowick, M., Cecka, C., Giomi, L., Middleton, A., Zielnicki, K., 2014. Thomson problem @ S. U.

URL http://thomson.phy.syr.edu/

[40] Bowick, M. J., Cacciuto, A., Nelson, D. R., Travesset, A., 2006. Crystalline particle packings on a sphere with long-range power-law potentials. Phys. Rev. B 73, 024115.

[41] Bowick, M. J., Giomi, L., 2009. Two-dimensional matter: order, curvature and defects. Advances in Physics 58 (5), 449-563.

[42] Brandolini, L., Choirat, C., Colzani, L., Gigante, G., Seri, R., Travaglini, G., 2013 (in press). Quadrature rules and distribution of points on manifolds. Ann. Sc. Norm. Super. Pisa Cl. Sci., 35.

[43] Brauchart, J. S., 2006. About the second term of the asymptotics for optimal Riesz energy on the sphere in the potential-theoretical case. Integral Transforms Spec. Funct. 17 (5), 321-328.

[44] Brauchart, J. S., 2008. Optimal logarithmic energy points on the unit sphere. Math. Comp. 77 (263), 1599-1613. 
[45] Brauchart, J. S., 2011. Optimal discrete Riesz energy and discrepancy. Unif. Distrib. Theory $6(2), 207-220$.

[46] Brauchart, J. S., 2011. A remark on exact formulas for the Riesz energy of the Nth roots of unity. arXiv:1105.5530v1 [math-ph].

[47] Brauchart, J. S., Dick, J., 2012. Quasi-Monte Carlo rules for numerical integration over the unit sphere $\mathbb{S}^{2}$. Numer. Math. 121 (3), 473-502.

[48] Brauchart, J. S., Dick, J., 2013. A Characterization of Sobolev Spaces on the Sphere and an Extension of Stolarsky's Invariance Principle to Arbitrary Smoothness. Constr. Approx. 38 (3), 397-445.

[49] Brauchart, J. S., Dick, J., 2013. A simple proof of Stolarsky's invariance principle. Proc. Amer. Math. Soc. 141 (6), 2085-2096.

[50] Brauchart, J. S., Dick, J., Fang, L., 2014. Spatial low-discrepancy sequences, spherical cone discrepancy, and applications in financial modeling, manuscript, arXiv:1408.4609.

[51] Brauchart, J. S., Dick, J., Saff, E. B., Sloan, I. H., Wang, Y. G., Womersley, R. S., 2014. Covering of spheres by spherical caps and worst-case error for equal weight cubature in Sobolev spaces. arXiv:1407.8311v1 [math.NA].

[52] Brauchart, J. S., Dragnev, P. D., Saff, E. B., 2009. Riesz extremal measures on the sphere for axis-supported external fields. J. Math. Anal. Appl. 356 (2), 769-792.

[53] Brauchart, J. S., Dragnev, P. D., Saff, E. B., 2014. Riesz external field problems on the hypersphere and optimal point separation. Potential Analysis, 1-32.

[54] Brauchart, J. S., Dragnev, P. D., Saff, E. B., van de Woestijne, C. E., 2012. A fascinating polynomial sequence arising from an electrostatics problem on the sphere. Acta Math. Hungar. 137 (1-2), 10-26.

[55] Brauchart, J. S., Hardin, D. P., Saff, E. B., 2007. The support of the limit distribution of optimal Riesz energy points on sets of revolution in $\mathbb{R}^{3}$. J. Math. Phys. 48 (12), 122901, 24.

[56] Brauchart, J. S., Hardin, D. P., Saff, E. B., 2009. Riesz energy and sets of revolution in $\mathbb{R}^{3}$. In: Functional analysis and complex analysis. Vol. 481 of Contemp. Math. Amer. Math. Soc., Providence, RI, pp. 47-57.

[57] Brauchart, J. S., Hardin, D. P., Saff, E. B., 2009. The Riesz energy of the Nth roots of unity: an asymptotic expansion for large N. Bull. Lond. Math. Soc. 41 (4), 621-633. 
[58] Brauchart, J. S., Hardin, D. P., Saff, E. B., 2012. Discrete energy asymptotics on a Riemannian circle. Unif. Distrib. Theory 7 (2), 77-108.

[59] Brauchart, J. S., Hardin, D. P., Saff, E. B., 2012. The next-order term for optimal Riesz and logarithmic energy asymptotics on the sphere. In: Recent advances in orthogonal polynomials, special functions, and their applications. Vol. 578 of Contemp. Math. Amer. Math. Soc., Providence, RI, pp. 31-61.

[60] Brauchart, J. S., Hesse, K., 2007. Numerical integration over spheres of arbitrary dimension. Constr. Approx. 25 (1), 41-71.

[61] Brauchart, J. S., Saff, E. B., Sloan, I. H., Womersley, R. S., 2014. QMC designs: optimal order quasi Monte Carlo integration schemes on the sphere. Math. Comp. 83, 2821-2851.

[62] Brauchart, J. S., Womersley, R. S., 2014. Weighted QMC designs: numerical integration on the unit sphere, $\mathbb{L}_{2}$ discrepancy and sums of distances, in preparation.

[63] Bresges, C., Urbanetz, N. A., 2008. Determination of the minimum number of spacer particles ensuring non-contact between host particles a new approach by numerical modelling. Powder Technology 187 (3), 260-272.

[64] Calef, M. T., 2009. Theoretical and computational investigations of minimal energy problems. Ph.D. thesis, Vanderbilt University.

[65] Calef, M. T., Griffiths, W., Schulz, A., Fichtl, C., Hardin, D. P., 2013. Observed asymptotic differences in energies of stable and minimal point configurations on $\mathbb{S}^{2}$ and the role of defects. J. Math. Phys. 54 (10), 101901, 20.

[66] Calef, M. T., Hardin, D. P., 2009. Riesz s-equilibrium measures on $d$-rectifiable sets as $s$ approaches $d$. Potential Anal. 30 (4), 385-401.

[67] Chen, X., Frommer, A., Lang, B., 2011. Computational existence proofs for spherical t-designs. Numer. Math. 117 (2), 289-305.

[68] Chen, X., Womersley, R. S., 2006. Existence of solutions to systems of underdetermined equations and spherical designs. SIAM J. Numer. Anal. 44 (6), 2326-2341 (electronic).

[69] Choirat, C., Seri, R., 2013. Computational aspects of Cui-Freeden statistics for equidistribution on the sphere. Math. Comp. 82 (284), 2137-2156.

[70] Choirat, C., Seri, R., 2013. Numerical properties of generalized discrepancies on spheres of arbitrary dimension. J. Complexity 29 (2), 216-235.

[71] Claeys, T., Kuijlaars, A. B. J., Vanlessen, M., 2008. Multi-critical unitary random matrix ensembles and the general Painlevé II equation. Ann. of Math. (2) 168 (2), 601-641. 
[72] Cohn, H., 2002. New upper bounds on sphere packings. II. Geom. Topol. 6, 329-353 (electronic).

[73] Cohn, H., Elkies, N., 2003. New upper bounds on sphere packings. I. Ann. of Math. (2) 157 (2), 689-714.

[74] Cohn, H., Kumar, A., 2007. Universally optimal distribution of points on spheres. J. Amer. Math. Soc. 20 (1), 99-148.

[75] Cohn, H., Kumar, A., 2009. Optimality and uniqueness of the Leech lattice among lattices. Ann. of Math. (2) 170 (3), 1003-1050.

[76] Cohn, H., Kumar, A., Schürmann, A., 2009. Ground states and formal duality relations in the Gaussian core model. Phys. Rev. E 80, 061116.

[77] Cohn, H., Woo, J., 2012. Three-point bounds for energy minimization. J. Amer. Math. Soc. 25 (4), 929-958.

[78] Conway, J. H., Sloane, N. J. A., 1993. Sphere packings, lattices and groups. Vol. 290 of Grundlehren der Mathematischen Wissenschaften [Fundamental Principles of Mathematical Sciences]. Springer-Verlag, New York.

[79] Coombs, D., Straube, R., Ward, M., 2009. Diffusion on a sphere with localized traps: Mean first passage time, eigenvalue asymptotics, and fekete points. SIAM Journal on Applied Mathematics 70 (1), 302-332.

[80] Coulangeon, R., 2006. Spherical designs and zeta functions of lattices. Int. Math. Res. Not., Art. ID 49620, 16.

[81] Coulangeon, R., Schürmann, A., 2012. Energy minimization, periodic sets and spherical designs. Int. Math. Res. Not. IMRN 2012 (4), 829-848.

[82] Cui, J., Freeden, W., 1997. Equidistribution on the sphere. SIAM J. Sci. Comput. 18 (2), 595-609.

[83] Damelin, S. B., Grabner, P. J., 2003. Energy functionals, numerical integration and asymptotic equidistribution on the sphere. J. Complexity 19, 231-246, corrigendum, 20 (2004), 883-884.

[84] Delsarte, P., Goethals, J. M., Seidel, J. J., 1977. Spherical codes and designs. Geometriae Dedicata 6 (3), 363-388.

[85] Dick, J., Pillichshammer, F., 2010. Digital Nets and Sequences. Discrepancy Theory and Quasi-Monte Carlo Integration. Cambridge University Press, Cambridge.

[86] Dragnev, P. D., Legg, D. A., Townsend, D. W., 2002. Discrete logarithmic energy on the sphere. Pacific J. Math. 207 (2), 345-358. 
[87] Dragnev, P. D., Saff, E. B., 2007. Riesz spherical potentials with external fields and minimal energy points separation. Potential Anal. 26 (2), 139-162.

[88] Erber, T., Hockney, G. M., 1997. Complex systems: equilibrium configurations of $N$ equal charges on a sphere $(2 \leq N \leq 112)$. In: Advances in chemical physics, Vol. XCVIII. Adv. Chem. Phys., XCVIII. Wiley, New York, pp. 495-594.

[89] Erdélyi, T., Saff, E. B., 2013. Riesz polarization inequalities in higher dimensions. J. Approx. Theory 171, 128-147.

[90] Farkas, B., Nagy, B., 2008. Transfinite diameter, Chebyshev constant and energy on locally compact spaces. Potential Anal. 28 (3), 241-260.

[91] Fejes Tóth, L., 1964. Regular figures. A Pergamon Press Book. The Macmillan Co., New York.

[92] Fejes Tóth, L., 1972. Lagerungen in der Ebene auf der Kugel und im Raum, 2nd Edition. Vol. 65 of Die Grundlehren der mathematischen Wissenschaften. SpringerVerlag, Berlin.

[93] Fekete, M., 1923. Über die Verteilung der Wurzeln bei gewissen algebraischen Gleichungen mit ganzzahligen Koeffizienten. Mathematische Zeitschrift 17 (1), 228-249.

[94] Feng, R., Zelditch, S., 2013. Random Riesz energies on compact Kähler manifolds. Trans. Amer. Math. Soc. 365 (10), 5579-5604.

[95] Forrester, P. J., 2010. Log-gases and random matrices. Vol. 34 of London Mathematical Society Monographs Series. Princeton University Press, Princeton, NJ.

[96] Fuselier, E. J., Wright, G. B., 2009. Stability and error estimates for vector field interpolation and decomposition on the sphere with RBFs. SIAM J. Numer. Anal. 47 (5), 3213-3239.

[97] Górski, K. M., Hivon, E., Banday, A. J., Wandelt, B. D., Hansen, F. K., Reinecke, M., Bartelmann, M., 2005. HEALPix: A framework for high-resolution discretization and fast analysis of data distributed on the sphere. The Astrophysical Journal 622 (2), 759.

[98] Götz, M., 2000. On the distribution of weighted extremal points on a surface in $\mathbf{R}^{d}, d \geq 3$. Potential Anal. 13 (4), 345-359.

[99] Götz, M., 2003. On the Riesz energy of measures. J. Approx. Theory 122 (1), 62-78.

[100] Götz, M., Saff, E. B., 2001. Note on $d$-extremal configurations for the sphere in $\mathbb{R}^{d+1}$. In: Recent progress in multivariate approximation (Witten-Bommerholz, 2000). Vol. 137 of Internat. Ser. Numer. Math. Birkhäuser, Basel, pp. 159-162. 
[101] Gourary, B. S., Adrian, F. J., 1960. Wave functions for electron-excess color centers in alkali halide crystals. In: Seitz, F., Turnball, D. (Eds.), Solid State Physics: Advances in Research and Applications. Vol. 10. Academic Press, New York and London, pp. $127-247$.

[102] Grabner, P. J., 1991. Erdős-Turán type discrepancy bounds. Monatsh. Math. 111, $127-135$.

[103] Grabner, P. J., 2014. Point sets of minimal energy. In: Larcher, G., Pillichshammer, F., Winterhof, A., Xing, C. (Eds.), Applications of Algebra and Number Theory. Cambridge University Press, pp. 104-117, essays in Honour of Harald Niederreiter.

[104] Grabner, P. J., Tichy, R. F., 1993. Spherical designs, discrepancy and numerical integration. Math. Comp. 60, 327-336.

[105] Gräf, M., 2013. Efficient algorithms for the computation of optimal quadrature points on riemannian manifolds. Ph.D. thesis, Technische Universität Chemnitz.

[106] Gräf, M., Potts, D., 2011. On the computation of spherical designs by a new optimization approach based on fast spherical Fourier transforms. Numer. Math. 119 (4), 699-724.

[107] Gräf, M., Potts, D., 2013. Table of spherical designs. Website.

URL http://www-user.tu-chemnitz.de/ potts/workgroup/graef/quadrature

[108] Gräf, M., Potts, D., Steidl, G., 2012. Quadrature errors, discrepancies, and their relations to halftoning on the torus and the sphere. SIAM Journal on Scientific Computing 34 (5), A2760-A2791.

[109] Habicht, W., van der Waerden, B. L., 1951. Lagerung von Punkten auf der Kugel. Math. Ann. 123, 223-234.

[110] Hales, T. C., 2005. A proof of the Kepler conjecture. Ann. of Math. (2) 162 (3), $1065-1185$.

[111] Harbrecht, H., Wendland, W. L., Zoriŭ, N. V., 2012. On Riesz minimal energy problems. J. Math. Anal. Appl. 393 (2), 397-412.

[112] Harbrecht, H., Wendland, W. L., Zoriŭ, N. V., 2014. Rapid solution of minimal Riesz energy problems. Preprint 2014-09, Mathematisches Institut, Universität Basel, Switzerland.

[113] Harbrecht, H., Wendland, W. L., Zoriŭ, N. V., 2014. Riesz minimal energy problems on $C^{k-1,1}$-manifolds. Math. Nachr. 287 (1), 48-69.

[114] Hardin, D. P., Kendall, A. P., Saff, E. B., 2013. Polarization optimality of equally spaced points on the circle for discrete potentials. Discrete Comput. Geom. 50 (1), $236-243$. 
[115] Hardin, D. P., Saff, E. B., 2004. Discretizing manifolds via minimum energy points. Notices Amer. Math. Soc. 51 (10), 1186-1194.

[116] Hardin, D. P., Saff, E. B., 2005. Minimal Riesz energy point configurations for rectifiable $d$-dimensional manifolds. Adv. Math. 193 (1), 174-204.

[117] Hardin, D. P., Saff, E. B., Simanek, B., 2014. Periodic discrete energy for long-range potentials, manuscript, arXiv:1403.7505v1 [math-ph].

[118] Hardin, D. P., Saff, E. B., Stahl, H., 2007. Support of the logarithmic equilibrium measure on sets of revolution in $\mathbb{R}^{3}$. J. Math. Phys. 48 (2), 022901, 14.

[119] Hardin, D. P., Saff, E. B., Whitehouse, J. T., 2012. Quasi-uniformity of minimal weighted energy points on compact metric spaces. Journal of Complexity 28 (2), $177-191$.

[120] Hardin, R. H., Sloane, N. J. A., 1993. A new approach to the construction of optimal designs. J. Statist. Plann. Inference 37 (3), 339-369.

[121] Hardin, R. H., Sloane, N. J. A., 1995. Codes (spherical) and designs (experimental). In: Different aspects of coding theory (San Francisco, CA, 1995). Vol. 50 of Proc. Sympos. Appl. Math. Amer. Math. Soc., Providence, RI, pp. 179-206.

[122] Hardin, R. H., Sloane, N. J. A., 1996. McLaren's improved snub cube and other new spherical designs in three dimensions. Discrete Comput. Geom. 15 (4), 429-441.

[123] Hardin, R. H., Sloane, N. J. A., 2002. Table of spherical designs. website, http://neilsloane.com/sphdesigns/dim3/.

[124] Hardin, R. H., Sloane, N. J. A., Smith, W. D., 1997. Minimal energy arrangements of points on a sphere. website, http://neilsloane.com/electrons/.

[125] Harman, G., 1982. Sums of distances between points of a sphere. Internat. J. Math. Math. Sci. 5 (4), 707-714.

[126] Hesse, K., 2006. A lower bound for the worst-case cubature error on spheres of arbitrary dimension. Numer. Math. 103 (3), 413-433.

[127] Hesse, K., Sloan, I. H., 2005. Optimal lower bounds for cubature error on the sphere $S^{2}$. J. Complexity 21 (6), 790-803.

[128] Hesse, K., Sloan, I. H., 2005. Worst-case errors in a Sobolev space setting for cubature over the sphere $S^{2}$. Bull. Austral. Math. Soc. 71 (1), 81-105.

[129] Hesse, K., Sloan, I. H., 2006. Cubature over the sphere $S^{2}$ in Sobolev spaces of arbitrary order. J. Approx. Theory 141 (2), 118-133. 
[130] Holhoş, A., Roşca, D., 2014. An octahedral equal area partition of the sphere and near optimal configurations of points. Comput. Math. Appl. 67 (5), 1092-1107.

[131] Hou, X., Shao, J., 2011. Spherical distribution of 5 points with maximal distance sum. Discrete Comput. Geom. 46 (1), 156-174.

[132] Korevaar, J., 1996. Fekete extreme points and related problems. In: Approximation theory and function series (Budapest, 1995). Vol. 5 of Bolyai Soc. Math. Stud. János Bolyai Math. Soc., Budapest, pp. 35-62.

[133] Korevaar, J., Meyers, J. L. H., 1993. Spherical Faraday cage for the case of equal point charges and Chebyshev-type quadrature on the sphere. Integral Transform. Spec. Funct. 1 (2), 105-117.

[134] Korevaar, J., Meyers, J. L. H., 1994. Chebyshev-type quadrature on multidimensional domains. J. Approx. Theory 79 (1), 144-164.

[135] Korobov, N. M., 1959. Approximate evaluation of repeated integrals. Dokl. Akad. Nauk SSSR 124, 1207-1210.

[136] Kuijlaars, A. B. J., Saff, E. B., 1998. Asymptotics for minimal discrete energy on the sphere. Trans. Amer. Math. Soc. 350 (2), 523-538.

[137] Kuipers, L., Niederreiter, H., 1974. Uniform distribution of sequences. WileyInterscience, New York.

[138] LaFave Jr., T., 2013. Correspondences between the classical electrostatic Thomson problem and atomic electronic structure. Journal of Electrostatics 71 (6), 1029-1035.

[139] LaFave Jr., T., 2014. Discrete transformations in the Thomson problem. Journal of Electrostatics 72 (1), 39-43.

[140] Landkof, N. S., 1972. Foundations of modern potential theory. Springer-Verlag, New York, translated from the Russian by A. P. Doohovskoy, Die Grundlehren der mathematischen Wissenschaften, Band 180.

[141] Le Gia, Q. T., Sloan, I. H., Wendland, H., 2010. Multiscale analysis in Sobolev spaces on the sphere. SIAM J. Numer. Anal. 48 (6), 2065-2090.

[142] Leopardi, P., 2006. A partition of the unit sphere into regions of equal area and small diameter. Electron. Trans. Numer. Anal. 25, 309-327 (electronic).

[143] Leopardi, P., 2013. Discrepancy, separation and riesz energy of finite point sets on the unit sphere. Adv. Comput. Math. 39 (1), 27-43.

[144] Li, X.-J., Vaaler, J. D., 1999. Some trigonometric extremal functions and the ErdősTurán type inequalities. Indiana Univ. Math. J. 48 (1), 183-236. 
[145] López García, A., Saff, E. B., 2010. Asymptotics of greedy energy points. Math. Comp. 79 (272), 2287-2316.

[146] Lubotzky, A., Phillips, R., Sarnak, P., 1986. Hecke operators and distributing points on the sphere. I. Comm. Pure Appl. Math. 39 (S, suppl.), S149-S186, frontiers of the mathematical sciences: 1985 (New York, 1985).

[147] Lubotzky, A., Phillips, R., Sarnak, P., 1987. Hecke operators and distributing points on $S^{2}$. II. Comm. Pure Appl. Math. 40 (4), 401-420.

[148] Lyubich, Y. I., Vaserstein, L. N., 1993. Isometric embeddings between classical Banach spaces, cubature formulas, and spherical designs. Geom. Dedicata 47 (3), 327362 .

[149] Magnus, W., Oberhettinger, F., Soni, R. P., 1966. Formulas and theorems for the special functions of mathematical physics. Vol. 52 of Grundlehren der mathematischen Wissenschaften. Springer-Verlag, third enlarged edition.

[150] Martínez-Finkelshtein, A., Maymeskul, V., Rakhmanov, E. A., Saff, E. B., 2004. Asymptotics for minimal discrete Riesz energy on curves in $\mathbb{R}^{d}$. Canad. J. Math. $56(3), 529-552$.

[151] Marzo, J., Ortega-Cerdà, J., 2010. Equidistribution of Fekete points on the sphere. Constr. Approx. 32 (3), 513-521.

[152] Mays, A., 2013. A real quaternion spherical ensemble of random matrices. J. Stat. Phys. 153 (1), 48-69.

[153] Melnyk, T. W., Knop, O., Smith, W. R., 1977. Extremal arrangements of points and unit charges on a sphere: equilibrium configurations revisited. Canad. J. Chem. 55 (10), 1745-1761.

[154] Mhaskar, H. N., Saff, E. B., 1985. Where does the sup norm of a weighted polynomial live? (A generalization of incomplete polynomials). Constr. Approx. 1 (1), 71-91.

[155] Montgomery, H. L., 1988. Minimal theta functions. Glasgow Math. J. 30 (1), 75-85.

[156] Müller, C., 1966. Spherical harmonics. Vol. 17 of Lecture Notes in Mathematics. Springer-Verlag, Berlin.

[157] Musin, O. R., 2008. The kissing number in four dimensions. Ann. of Math. (2) 168 (1), $1-32$.

[158] Nebe, G., 2013. Boris Venkov's theory of lattices and spherical designs. In: Diophantine methods, lattices, and arithmetic theory of quadratic forms. Vol. 587 of Contemp. Math. Amer. Math. Soc., Providence, RI, pp. 1-19. 
[159] Nebe, G., Venkov, B., 2009. On lattices whose minimal vectors form a 6-design. European J. Combin. 30 (3), 716-724.

[160] Nerattini, R., Brauchart, J., Kiessling, M.-H., 2014. Optimal N-Point Configurations on the Sphere: "Magic" Numbers and Smales 7th Problem. Journal of Statistical Physics, 1-69.

[161] Nodari, S. R., Serfaty, S., 2014. Renormalized energy equidistribution and local charge balance in $2 \mathrm{~d}$ coulomb systems. International Mathematics Research Notices.

[162] Novak, E., Woźniakowski, H., 2008. Tractability of multivariate problems. Vol. 1: Linear information. Vol. 6 of EMS Tracts in Mathematics. European Mathematical Society (EMS), Zürich.

[163] Of, G., Wendland, W. L., Zoriŭ, N. V., 2010. On the numerical solution of minimal energy problems. Complex Var. Elliptic Equ. 55 (11), 991-1012.

[164] Pólya, G., Szegö, G., 1931. Über den transfiniten Durchmesser (Kapazitätskonstante) von ebenen und räumlichen Punktmengen. J. Reine Angew. Math. 165, 4-49.

[165] Pritsker, I. E., 2011. Distribution of point charges with small discrete energy. Proc. Amer. Math. Soc. 139 (10), 3461-3473.

[166] Pritsker, I. E., Saff, E. B., Wise, W., 2014. Reverse triangle inequalities for Riesz potentials and connections with polarization. J. Math. Anal. Appl. 410 (2), 868-881.

[167] Rakhmanov, E. A., Saff, E. B., Zhou, Y. M., 1994. Minimal discrete energy on the sphere. Math. Res. Lett. 1 (6), 647-662.

[168] Reimer, M., 2000. Hyperinterpolation on the sphere at the minimal projection order. J. Approx. Theory 104 (2), 272-286.

[169] Reimer, M., 2001. The geometry of nodes in a positive quadrature on the sphere. In: Recent progress in multivariate approximation (Witten-Bommerholz, 2000). Vol. 137 of Internat. Ser. Numer. Math. Birkhäuser, Basel, pp. 245-248.

[170] Reimer, M., 2003. Multivariate polynomial approximation. Vol. 144 of International Series of Numerical Mathematics. Birkhäuser Verlag, Basel.

[171] Rougerie, N., Serfaty, S., 2013. Higher dimensional Coulomb gases and renormalized energy functionals, manuscript, arXiv:1307.2805v3 [math-ph].

[172] Sadoc, J.-F., Mosseri, R., 1999. Geometrical frustration. Collection Aléa-Saclay: Monographs and Texts in Statistical Physics. Cambridge University Press, Cambridge.

[173] Saff, E. B., Kuijlaars, A. B. J., 1997. Distributing many points on a sphere. Math. Intelligencer 19 (1), 5-11. 
[174] Saff, E. B., Totik, V., 1997. Logarithmic potentials with external fields. Vol. 316 of Grundlehren der Mathematischen Wissenschaften [Fundamental Principles of Mathematical Sciences]. Springer-Verlag, Berlin, appendix B by Thomas Bloom.

[175] Sandier, E., Serfaty, S., 2012. From the Ginzburg-Landau model to vortex lattice problems. Comm. Math. Phys. 313 (3), 635-743.

[176] Sarnak, P., Strömbergsson, A., 2006. Minima of Epstein's zeta function and heights of flat tori. Invent. Math. 165 (1), 115-151.

[177] Schaback, R., 1995. Error estimates and condition numbers for radial basis function interpolation. Adv. Comput. Math. 3 (3), 251-264.

[178] Schoenberg, I. J., 1938. Metric spaces and positive definite functions. Trans. Amer. Math. Soc. 44 (3), 522-536.

[179] Schwartz, R. E., 2013. The five-electron case of Thomsons problem. Experimental Mathematics 22 (2), 157-186.

[180] Serfaty, S., 2014. Ginzburg-Landau Vortices, Coulomb Gases, and Renormalized Energies. J. Stat. Phys. 154 (3), 660-680.

[181] Seymour, P. D., Zaslavsky, T., 1984. Averaging sets: a generalization of mean values and spherical designs. Adv. in Math. 52 (3), 213-240.

[182] Shub, M., Smale, S., 1993. Complexity of Bezout's theorem. I: Geometric aspects. J. Am. Math. Soc. 6 (2), 459-501.

[183] Shub, M., Smale, S., 1993. Complexity of Bezout's theorem. III. Condition number and packing. J. Complexity 9 (1), 4-14, festschrift for Joseph F. Traub, Part I.

[184] Sloan, I. H., Womersley, R. S., 2004. Extremal systems of points and numerical integration on the sphere. Adv. Comput. Math. 21 (1-2), 107-125.

[185] Sloan, I. H., Womersley, R. S., 2009. A variational characterisation of spherical designs. J. Approx. Theory 159 (2), 308-318.

[186] Smale, S., 1998. Mathematical problems for the next century. Math. Intelligencer 20 (2), 7-15.

[187] Stolarsky, K. B., 1972. Sums of distances between points on a sphere. Proc. Amer. Math. Soc. 35, 547-549.

[188] Stolarsky, K. B., 1973. Sums of distances between points on a sphere. II. Proc. Amer. Math. Soc. 41, 575-582. 
[189] Tammes, P. M. L., 1930. On the origin of number and arrangement of the places of exit on the surface of pollen grains. Recueil des travaux botaniques néerlandais 27 , $1-84$.

[190] Teuber, T., Steidl, G., Gwosdek, P., Schmaltz, C., Weickert, J., 2011. Dithering by differences of convex functions. SIAM J. Imaging Sci. 4 (1), 79-108.

[191] Thomson, J. J., 1904. On the structure of the atom: an investigation of the stability and periods of oscillation of a number of corpuscles arranged at equal intervals around the circumference of a circle; with application of the results to the theory of atomic structure. Philos. Mag. 7 (39), 237-265.

[192] Thue, A., 1910. Über die dichteste Zusammenstellung von kongruenten Kreisen in einer Ebene. Christiania Vid.-Selsk. Skr. 1, 9p.

[193] Torquato, S., Stillinger, F. H., 2006. New conjectural lower bounds on the optimal density of sphere packings. Experiment. Math. 15 (3), 307-331.

[194] Tumanov, A., 2013. Minimal biquadratic energy of five particles on a 2-sphere. Indiana Univ. Math. J. 62 (6), 1717-1731.

[195] Venkov, B. B., 1984. Even unimodular extremal lattices. Trudy Mat. Inst. Steklov. 165, 43-48, algebraic geometry and its applications.

[196] Wagner, G., 1990. On means of distances on the surface of a sphere (lower bounds). Pacific J. Math. 144 (2), 389-398.

[197] Wagner, G., 1992. On means of distances on the surface of a sphere. II. Upper bounds. Pacific J. Math. 154 (2), 381-396.

[198] Wales, D. J., Doye, J. P. K., Dullweber, A., Hodges, M. P., Naumkin, F. Y., Calvo, F., Hernández-Rojas, J., Middleton, T. F., 2014. The Cambridge Cluster Database. URL http://www-wales.ch.cam.ac.uk/CCD .html

[199] Yudin, V. A., 1997. Lower bounds for spherical designs. Izv. Ross. Akad. Nauk Ser. Mat. 61 (3), 213-223.

[200] Zoriŭ, N. V., 2003. Equilibrium potentials with external fields. Ukraïn. Mat. Zh. 55 (9), 1178-1195.

[201] Zoriǔ, N. V., 2004. Potential theory with respect to consistent kernels: a completeness theorem, and sequences of potentials. Ukraïn. Mat. Zh. 56 (11), 1513-1526. 